\title{
Monte Carlo Simulations for RHIC Spin Physics
}

\author{
Stefan Güllenstern \\ Max-Planck-Institut for Nuclear Physics, P.O. Box 103980 \\ 69029 Heidelberg, Germany \\ Paweł Górnicki \\ Institute of Physics and Center for Theoretical Physics \\ Polish Academy of Sciences \\ Al. Lotników 32/46, 02-668 Warsaw, Poland \\ Lech Mankiewicz \\ Nicolaus Copernicus Astronomical Center, ul. Bartycka 18 \\ PL-00 716 Warsaw, Poland \\ Andreas Schäfer \\ Institute for Theoretical Physics, University Frankfurt \\ D-60054 Frankfurt am Main, Germany
}




\begin{abstract}
Direct photon production in longitudinally polarised proton-proton collisions offers the most direct and unproblematic possibility to determine the polarised gluon distribution of a proton. This information could play a major role for improving our understanding of the nucleon structure and QCD in general. It is hoped that such experiments will be done at RHIC. We present results of detailed Monte Carlo simulations using a code called SPHINX. We find that for RHIC energies and large gluon polarisation the Compton graph dominates allowing for a direct test of $\Delta g$. Triggering on away-side jets with the envisaged jet-criteria should allow to obtain more detailed information on $\Delta g(x)$. The photon asymmetry resulting from the asymmetry of produced $\pi^{0}$ 's provides an additional signal, which is complementary to the other two. For small gluon polarisation, i.e. $\Delta g \leq 0.5$ or very soft polarised gluon-distributions the envisaged experiments will require a highly sophisticated simulation and large statistics to extract more than upper bounds for $|\Delta g(x)|$.
\end{abstract}

PACS-No.: $\quad$ 13.88.+e, 13.85.Hd, 12.38.Bx 


\section{Introduction}

The experiments on longitudinally polarised lepton-nucleon scattering performed over the last years at CERN [1, 2] and SLAC [3] have determined the polarised structure function $g_{1}^{p}(x)$ with rather good accuracy, while $g_{1}^{n}(x)$ still needs improvement. The theoretical interpretation of these data is the subject of intense debates which we do not want to review here. These discussions have, however, established a number of facts which imply the importance of analysing polarised proton-proton collisions:

1.) Polarized reactions provide very sensitive tests of QCD. To realize this potential fully requires, however, a detailed experimental knowledge and theoretical understanding of $Q^{2}$-dependences, just as for unpolarised reactions [4. To disentangle the various effects precise and complementary measurements will be needed. In addition comparing specific results from deep-inelastic scattering and protonproton collisions allows for interesting tests. [5]

2.) The polarised gluon distribution plays a very special role as it contributes via the anomaly [6] in the same way as the polarised quark distribution. For deep inelastic scattering there is no way to distinguish between a 'genuine' quark distribution and an 'anomalous' gluon distribution. Such a distinction is by principle only possible by combining inclusive and exclusive data, see e. g. [8] for polarised deepinelastic scattering, though probably very difficult in praxis. On the other hand to establish the existence of an anomalous gluon contribution would test fundamental topological properties of QCD, which have never been directly accessible before.

Obviously in this situation a precise knowledge of $\Delta g\left(x, Q^{2}\right)$ would help tremendously, and this could be provided by polarised protonproton scattering. Such experiments would be possible at RHIC in the near future, details can be found in ref. [9].

Actually there are many interesting quantities which can be determined in such experiments, also for transverse polarisation, but their theoretical understanding is at least partially incomplete and the reliable simulation of background reactions will require fundamentally modified codes. We therefore concentrated on the measurement of $\Delta g$ via direct photon assymmetries, which is the most straight forward experiment proposed so far [10].

Still it is generally accepted (and will be demonstrated in this con- 
tribution) that planning and analysing such experiments requires a full-fledged Monte-Carlo code. We developped one such code to describe the collision of longitudinally polarised nucleons. It is called SPHINX and is basically a polarised version of PYTHIA [11]. A description of this code will be published elsewhere [12], and we shall try to make it generally accessible. In our paper we present results obtained with this code for experiments planned at RHIC using the STAR and/or PHENIX detector.

We present our results in three sections. In section 2 we discuss prompt- $\gamma$ production per se. In section 3 we analyse the additional information which is obtained by simultaneously detecting the awayside-jet, and in section 4 we present the photon asymmetry generated by an asymmetry in the production of $\pi^{0}$ 's subsequently decaying into photons.

\section{Prompt- $\gamma$-Production}

The goal of the measurement of prompt- $\gamma$-production at RHIC is the determination of the gluon polarisation $\Delta g$. For this it has to be clarified that the signal is indeed proportional to $\Delta g$ and can be clearly separated from the background. For this purpose we investigated the two leading processes (i.e. first order in $\alpha_{s}$ ) for prompt- $\gamma$-production, namely the Compton process (see figure 1) and the annihilation process (figure 2) and determined their contribution at RHIC energies to the cross section for different parton parametrisations. Actually the hard matrix elements for prompt- $\gamma$ production have been calculated to NLO [13], but PYTHIA is set up in such a way that the higher orders are effectively taken care of by the initial and final state showering. As the NLO amplitudes do not show any features qualitatively different from those of the LO ones this procedure should be fine. Note however, that for heavy-quark production the spin-effects could change substantially between LO and NLO [14], which would require a more carefull treatment.

Furthermore we had a closer look at the main contributions to the background and analysed the procedures proposed in [9] to discriminate it. 


\subsection{The Compton process vs. the annihilation process}

The hadronic cross section for prompt- $\gamma$-production in proton-proton collisions is given by a convolution of the parton distributions and the partonic cross section. In the spin averaged case $\left(\sigma=\frac{1}{2}(\sigma(\uparrow \downarrow)+\sigma(\uparrow \uparrow))\right.$, where $\uparrow \downarrow(\uparrow \uparrow)$ denotes antiparallel (parallel) spins of the two protons) it reads

$$
\begin{aligned}
& E_{\gamma} \frac{\mathrm{d} \sigma_{p p \rightarrow \gamma X}}{\mathrm{~d}^{3} p_{\gamma}}\left(s, x_{F}, p_{\perp}\right) \\
= & \sum_{a b} \int \mathrm{d} x_{a} \mathrm{~d} x_{b} P_{a}\left(x_{a}, Q^{2}\right) P_{b}\left(x_{b}, Q^{2}\right) E_{\gamma} \frac{\mathrm{d} \hat{\sigma}_{a b \rightarrow \gamma X}}{\mathrm{~d}^{3} p_{\gamma}}\left(\hat{s}, x_{F}, p_{\perp}\right)
\end{aligned}
$$

while for the spin difference $\left(\Delta \sigma=\frac{1}{2}(\sigma(\uparrow \downarrow)-\sigma(\uparrow \uparrow))\right)$ it is given by

$$
\begin{aligned}
& E_{\gamma} \frac{\mathrm{d} \Delta \sigma_{p p \rightarrow \gamma X}}{\mathrm{~d}^{3} p_{\gamma}}\left(s, x_{F}, p_{\perp}\right) \\
= & \sum_{a b} \int \mathrm{d} x_{a} \mathrm{~d} x_{b} \Delta P_{a}\left(x_{a}, Q^{2}\right) \Delta P_{b}\left(x_{b}, Q^{2}\right) E_{\gamma} \frac{\mathrm{d} \Delta \hat{\sigma}_{a b \rightarrow \gamma X}}{\mathrm{~d}^{3} p_{\gamma}}\left(\hat{s}, x_{F}, p_{\perp}\right)
\end{aligned}
$$

Here the sum is over all partonic subprocesses which contributes to the reaction $p p \rightarrow \gamma X . \quad P_{a}$ and $P_{b}$ denotes the unpolarised parton distributions of quarks and gluons, $\Delta P_{a}$ and $\Delta P_{b}$ the polarised ones. The latter are the difference between partons of the same helicity as the hadron and those of opposite helicity, the former are the sum of the two helicities. The partonic cross sections in the helicity averaged case is defined as $\hat{\sigma}=\frac{1}{2}\left(\hat{\sigma}_{++}+\hat{\sigma}_{+-}\right)$where the indices,+- signifies the helicities of the incoming partons. For $\Delta \hat{\sigma}$ the plus sign between the two terms in the formula above has to be replaced by a minus sign. $x_{a}$ and $x_{b}$ are the Bjørken $x$-variables of the incoming partons, $p_{\perp}$ is the transverse momentum of the outgoing photon in the $p p$-CMS. The longitudinal momentum fraction of the $\gamma$ is defined as $x_{F}=2 p_{\gamma}^{z} / \sqrt{s}$. The polarised partonic cross section is given by

$$
E_{\gamma} \frac{\mathrm{d} \Delta \hat{\sigma}_{a b \rightarrow \gamma X}}{\mathrm{~d}^{3} p_{\gamma}}=\alpha \alpha_{s} \frac{1}{\hat{s}}\left|\Delta \bar{M}_{a b \rightarrow \gamma X}\right|^{2} \delta(\hat{s}+\hat{t}+\hat{u}) .
$$

To obtain the unpolarised cross section one has simply to replace the polarised matrix element $\Delta \bar{M}_{a b \rightarrow \gamma X}$ by the unpolarised $\bar{M}_{a b \rightarrow \gamma X}$. 
Finally the partonic Mandelstam variables are related to the usual hadronic ones by: $\hat{s}=x_{a} x_{b} s, \hat{t}=x_{a} t$, and $\hat{u}=x_{b} u$.

In leading order perturbation theory in $\alpha \alpha_{s}$ only the Compton process $q g \rightarrow \gamma q$ and the annihilation process $q \bar{q} \rightarrow \gamma g$ contribute to the prompt- $\gamma$-production. Their polarised and unpolarised matrix elements are summarised in table 1 . The contribution of the two processes to the hadronic cross section corresponding to (11) resp. (2) depends strongly on the parton distributions. In unpolarised pp-collisions - in contrary to $p \bar{p}$-collisions - the Compton process clearly dominates over the annihilation process, because the gluon density in protons is much higher than the antiquark density $(g \gg \bar{q})$. In the polarised case, however, the relative importance of the two processes depends crucially on the relative size of the polarised gluon distribution $\Delta g$ and the polarised sea distribution $\Delta \bar{q}$. Both of them are presently completely unknown. By the time the RHIC-Spin-Collaboration (RSC) could possibly start to take data, $\Delta \bar{q}(x)$ should, however, be known quite accurately from semi-inclusive lepton-nucleon scattering experiments by the HERMES collaboration (HERA) 115]. This should allow to avoid ambiguities in the interpretation of potential RSC data.

For our simulation we used two parametrisations for parton densities with large gluon polarisation by Altarelli\&Stirling [16] and by Ross\&Roberts (set D) [17] and one parametrisation with a small gluon polarisation by Ross\&Roberts (set A).

For large gluon polarisation the Compton process is the by far dominant one and one can safely neglect the contribution of the annihilation process in (2). In this case the prompt- $\gamma$-production becomes proportional to $\Delta g$ and is such a clean probe for the gluon polarisation:

$$
\begin{aligned}
& E_{\gamma} \frac{\mathrm{d} \Delta \sigma_{p p \rightarrow \gamma X}}{\mathrm{~d}^{3} p_{\gamma}}\left(s, x_{F}, p_{\perp}\right) \\
\approx & \sum_{q} \int \mathrm{d} x_{a} \mathrm{~d} x_{b}\left(\Delta q\left(x_{a}, Q^{2}\right) \Delta g\left(x_{b}, Q^{2}\right) E_{\gamma} \frac{\mathrm{d} \Delta \hat{\sigma}_{q g \rightarrow \gamma q}}{\mathrm{~d}^{3} p_{\gamma}}\left(\hat{s}, x_{F}, p_{\perp}\right)+\left(x_{a} \leftrightarrow x_{b}\right)\right) .
\end{aligned}
$$

However, in a scenario with a large sea contribution to the spin of the proton and a gluon polarisation only due to Altarelli-Parisi evolution, as described by the parametrisation Ross\&Roberts set A [17], the annihilation process becomes the major contribution.

We investigated these different scenarios with the Monte-Carlo program SpHINx, which can be used to simulate longitudinal polarised 
$p p$-scattering. We generated $10^{7}$ events for both spin combinations of the protons at the RHIC energy $\sqrt{s}=200 \mathrm{GeV}$. For the unpolarised parton distributions we haven chosen the parametrisation of Glück, Reya, and Vogt [21], while for the polarised distributions we used the parametrisations mentioned above, namely Altarelli\&Stirling and Ross\&Roberts set A and set D. In Sphinx matrix elements are implemented in leading order only. However, due to the initial and final state shower algorithm some features of higher order effects are incorporated as well [11]. Also the polarisation effects are traced in the initial state shower. For the simulations the polarised initial state shower and the final state shower were switched on. To avoid infrared divergences the hard interaction cross section must be supplemented by a lower cut off for the transverse momentum $p_{\perp}$. We chose $p_{\perp} \geq 4 \mathrm{GeV}$.

The results of these simulations are shown in figures 3 to 21 . In figure 3 the Lorentz-invariant cross section for prompt- $\gamma$-production as a function of $p_{\perp}$ at $x_{F} \approx 0$ is displayed for the Compton process (upper plot) and the annihilation process (lower plot). $x_{F}$ is here the longitudinal momentum fraction of the photon defined by $x_{F}=$ $2 p_{z}^{\gamma} / \sqrt{s}$. In both cases the spin averaged cross section (squares) and the cross section for the spin difference (triangles) are shown. For the latter the parametrisation of Altarelli\&Stirling has been used. For the annihilation process $-\Delta \sigma$ is plotted, because $\Delta \sigma$ is negative, meaning that the cross-section for antiparallel spins is smaller than for parallel spin. This can be seen from table 1 , keeping in mind that $\hat{t}=x_{1} x_{3} t=$ $-\hat{s}(1-\cos \theta) / 2$ is negative. This negative polarised partonic cross section is than multiplied by the positve polarised quark and antiquark distributions in (2). The error bars reflect the MC error. A typical RHIC run has $320 \mathrm{pb}^{-1}$, such that the $10^{7}$ events we generated for each spin combination (with $p_{\perp} \geq 4 \mathrm{GeV}$ ) corresponds to an integrated cross section of $3 \cdot 10^{-5} \mathrm{mb}$ for the spin averaged case respectively to a differential cross-section of roughly $3 \cdot 10^{-5} \mathrm{mb} /(4 \pi \cdot 4 \mathrm{GeV})=6 \cdot 10^{-7}$ $\mathrm{mb} / \mathrm{GeV}$ in the $4 \mathrm{GeV}$ bin. This implies that the MC error is roughly comparable to the expected experimental error for the prompt- $\gamma$ 's and substantially larger than the anticipated experimental errors for gammas from $\pi^{0}$ decays.

At this point we want to state as clearly as possible that we do not attribute special significance to any of the used parametrisations. In fact virtually nothing is known about $\Delta g\left(x, Q^{2}\right)$, except the trivial 
fact that its absolute magnitude is limited by $g\left(x, Q^{2}\right)$. It could e.g. very well be that $\Delta g\left(x, Q^{2}\right)$ changes sign for some $x$ value and this was actually advocated to get a good fit to the data in specific models. It is just because nothing is known about $\Delta g\left(x, Q^{2}\right)$ that it is so important to measure (and RHIC seems to be the only facility able to do so). In this situation all our simulations are meant as illustrations. We used two similar models (Altarelli\&Stirling and Ross\&Roberts D) with optimistically large gluon-distributions, because it is easy to estimate the results for smaller $\Delta g$ by just scaling them down and for large $\Delta g$ the MC simulation requires less statistics. At the same time the difference between the predictions of these two models gives a feeling for the sensitivity of experimental signals to details of the distribution functions.

Comparing the two processes one can clearly see that in the spin averaged case the Compton process leads to a cross section by an order of magnitude larger than the annihilation process. This domination is even more pronounced in the polarised case, where one and a half orders of magnitude are between both processes. However, the latter fact is, as said above, due to the choice of a parametrisation with a large $\Delta g$ and a small $\Delta \bar{q}$.

In figure 1 we examine the polarised case in more detail by using different sets of polarised parton distributions. In the upper part the cross section of the spin difference for the Compton process for three different sets of parametrisations is shown. The parametrisations of Altarelli\&Stirling (triangles) and Ross\&Roberts set D (squares) have a similar, large $\Delta g$, but the $x$-dependence $\Delta g(x)$ is different, which leads to much bigger cross section (logarithmic scale!) in the latter case in comparison with the former. This means that it is possible to distinguish between both parametrisations in the experiment. The third parametrisation Ross\&Roberts set A (circles) has a gluon polarisation only due to Altarelli-Parisi evolution and leads to no measurable signal.

In the lower part the annihilation process is shown. Here the results for Altarelli\&Stirling (triangles), as a representative of the large polarised gluons and small polarised sea scenario, and Ross\&Roberts set A (circles), as a representative of the opposite situation of nearly unpolarised gluons and large, negative polarised sea, are given. As seen above, Altarelli\&Stirling leads to a small, negative signal, whereas now Ross\&Roberts set A produce a relatively large, positive signal, which 
exceeds the corresponding result for the Compton process by far. On the other hand it is still by roughly a factor of five smaller than the result of Compton process in case of a large gluon polarisation.

In figure 5 the resulting asymmetries $\Delta \sigma / \sigma$ are shown. For the Compton process in the upper part the asymmetry grows with $p_{\perp}$ for Altarelli\&Stirling and Ross\&Roberts set D and reaches values around $20 \%$ resp. $30 \%$. For Ross\&Roberts set A it is consistent with zero. For the annihilation process in the lower part Altarelli\&Stirling leads to small, negative, Ross\&Roberts set A to a large, positive asymmetry.

The main conclusions one can draw at this point are that in the case of a large gluon polarisation the annihilation process is a small correction, which is calculable using the data from electron-scattering, such that prompt- $\gamma$-production is a clean probe of $\Delta g$. If the absolute value of $\Delta g(x)$ would be a factor ten smaller than assumed by e.g. Altarelli\&Stirling, which would be the case if the total spin carried by gluons were less than half a unit of $\hbar$, then annihilation and Compton graph would contribute at the same level. It would still be possible to extract $\Delta g\left(x, Q^{2}\right)$, but only by a combined fit to the data from various spin-experiments. If $\Delta g$ would be substantially smaller than $0.5 \hbar$ the determination of $\Delta g\left(x, Q^{2}\right)$ were probably very difficult (unless rather large positive and negative parts of $\Delta g(x)$ canceled to give a small $\Delta g$ ). However, deriving such a low bound for $\Delta g(x)$ would be very interesting as one would not expect it to be that small. The crucial remaining questions are whether the anticipated statistics is sufficent to actually determine $\Delta g(x)$ and whether there are background effects which could blur the simple picture. These questions will be addressed next.

To examine the precission of prompt- $\gamma$ measurements at RHIC, we transformed these cross sections in counting rates at RHIC and determined the statistical errors. To obtain the total rates one has to multiply the cross section with the integrated luminosity for which we have taken the design value from [9]: $\int \mathcal{L} \mathrm{d} \tau=3.2 \times 10^{38} \mathrm{~cm}^{-2}$, which corresponds to a luminosity of $\mathcal{L}=8 \times 10^{31} \mathrm{~cm}^{-2} \mathrm{~s}^{-1}$ and an effective run time of $\tau=4 \times 10^{6} \mathrm{~s}$, which means 100 days with $50 \%$ efficiency. At RHIC the beams are only partially polarised: $P_{\text {beam }}=0.7$, whereas Sphinx simulates fully polarised events. Therefore one has to combine the MC-rates to the experimental rates as follows $\left(P=P_{\text {beam }} \times P_{\text {beam }}\right)$ :

$$
N_{\mathrm{exp} .}^{\uparrow \downarrow}=\frac{1+P}{4} N_{\mathrm{MC}}^{\uparrow \downarrow}+\frac{1-P}{4} N_{\mathrm{MC}}^{\uparrow \uparrow}
$$




$$
N_{\mathrm{exp} .}^{\uparrow \uparrow}=\frac{1+P}{4} N_{\mathrm{MC}}^{\uparrow \uparrow}+\frac{1-P}{4} N_{\mathrm{MC}}^{\uparrow \downarrow}
$$

For the asymmetry follows:

$$
\begin{aligned}
A_{\text {exp. }} & \equiv \frac{N_{\text {exp. }}^{\uparrow \downarrow}-N_{\text {exp. }}^{\uparrow \downarrow}}{N_{\text {exp. }}^{\uparrow \downarrow}+N_{\text {exp }}^{\uparrow \downarrow} .} \\
& =P \frac{N_{\mathrm{MC}}^{\uparrow \downarrow}-N_{\mathrm{MC}}^{\uparrow \downarrow}}{N_{\mathrm{MC}}^{\uparrow \downarrow}+N_{\mathrm{MC}}^{\uparrow \downarrow}} \\
& \equiv P A_{\mathrm{MC}},
\end{aligned}
$$

with the error:

$$
\begin{aligned}
\delta A_{\text {exp. }} & =\frac{2 \sqrt{N_{\text {exp. }}^{\uparrow \downarrow} N_{\text {exp. }}^{\uparrow \uparrow}}}{N_{\text {exp. }}^{\uparrow \downarrow}+N_{\text {exp. }}^{\uparrow \uparrow}} \frac{1}{\sqrt{N_{\text {exp. }}^{\uparrow \downarrow}+N_{\text {exp. }}^{\uparrow \uparrow}}} \\
& \approx \frac{1}{\sqrt{N_{\text {exp. }}^{\uparrow \downarrow}+N_{\text {exp }}^{\uparrow \uparrow}}} .
\end{aligned}
$$

At RHIC several cuts on the events have to be applied. One cut, which we have investigated further, is due to the finite coverage in the plane of the pseudorapidity $\eta$ and the azimuth $\phi$ of the detectors STAR and PHENIX [9]. STAR has a full coverage in the azimuth: $\Delta \phi=2 \pi$ and a coverage in the pseudorapidity of $|\eta|<1$ without end caps and of $|\eta|<2$ in the extended version with end caps. PHENIX covers only the half azimuth $\Delta \phi=\pi$ and has the same $\eta$-coverage as STAR. To examine how severe these cuts are we determined the rates for prompt- $\gamma$-production assuming a full azimuthal coverag $€ \square$ and applying the cuts $\left|\eta^{\gamma}\right|<1,\left|\eta^{\gamma}\right|<2$, and no $\eta^{\gamma}$-cut. The results are given in the figures 6, 6. The error bars reflect the statistical errors at RHIC.

In figure 6 the spin averaged rates (upper left plot), the spin difference rates (upper right plot), and the asymmetry (lower plot) are displayed for the three $\eta^{\gamma}$-cuts. The parametrisation of Altarelli\&Stirling has been used. One can clearly see that the cut $\left|\eta^{\gamma}\right|<2$ is not very severe, especially for high $p_{\perp}$, whereas the cut $\left|\eta^{\gamma}\right|<1$ reduces the rates roughly by a factor of two. In addition also the asymmetry decreases

\footnotetext{
${ }^{1}$ This means that we are simulating the situation for STAR. To obtain the corresponding results for PHENIX one has to divide the rates by 2 .
} 
in this case slightly. However, even with the stronger cut $\left|\eta^{\gamma}\right|<1$ similar parametrisations as Altarelli\&Stirling and Ross\&Roberts set D can be distinguished within the experimental errors at RHIC, as can be seen in figure 7. Here the rates for the spin difference and the asymmetries for the different parametrisations in dependence of the applied cuts are compared. Hence at this point the extended version of detectors with end caps is not absolutely needed, but, as shown later, this extension will be crucial when measuring the prompt- $\gamma$ and the away-side jet.

\subsection{Background considerations}

High- $p_{\perp} \gamma$ 's are not only produced in the direct processes discussed in the subsection above, but at a far larger rate due to bremsstrahlung and in particular in meson decays. This background has to be separated from the direct photons very accurately in order to do not contaminate the signal substantially. In this publication we concentrate on the background produced in pion and $\eta$ decays $\pi^{0} \rightarrow 2 \gamma$ resp. $\eta \rightarrow 2 \gamma$ which is the major contribution and analyse the ability to remove it with STAR and PHENIX.

The mesons which give rise to the background are produced in all QCD parton processes, among them the QCD-Compton process:

$$
\begin{aligned}
& q g \rightarrow g q \longrightarrow \ldots \pi^{0} \rightarrow \ldots \gamma \gamma \\
& q g \rightarrow g q \longrightarrow \ldots \eta \rightarrow \ldots \gamma \gamma
\end{aligned}
$$

is the most important, followed by $q q \rightarrow q q$ and $g g \rightarrow g g$. Beside the QCD-Compton process we had a closer look at the $g g$-scattering process, because this process is especially interesting with regard to the gluon polarisation. Here $\Delta g$ enters twice in the cross section, and hence this process is extremly sensitive on changes in $\Delta g$. For our studies we simulated $10^{7}$ events for each polarisation combination of the protons for both, the QCD-Compton process $q g \rightarrow g q$ and the $g g$-scattering process $g g \rightarrow g g$.

Comparing the yields of the QCD-Compton process (full symbols) with the prompt- $\gamma$ Compton process (open symbols) in figure 8 it is obvious that the background is very important in the case of a large gluon polarisation (Altarelli\&Stirling left, Ross\&Roberts right). In the spin averaged case (upper plots) it is much larger than the signal, but it decreases also faster with $p_{\perp}$. In addition, the background 
shows also an asymmetry and the resulting rates for the spin-difference (lower plots) are higher than the true signal up to a transverse momentum of $p_{\perp} \approx 15 \mathrm{GeV}$. Because the QCD-Compton process is just one contribution, although the most important, to the background, it is obvious that the photons from neutral meson decay have to be separated very accurately from the direct ones in order not to contaminate the true signal. Doing so has the additional advantage that the asymmetry in the photons from e.g. pion decay can be used as an excellent signal for $\Delta g(x)$ (see section 4 ).

To investigate the background further let us consider how it is composed. Figure 9 and 10 show the compositon for the QCD-Compton process and the $g g$-scattering process. In both cases the the pion decay $\pi^{0} \rightarrow 2 \gamma$ give rise to the main contribution of $80 \%-90 \%$ (logarithmic scale!) over the whole $p_{\perp}$-region for both, the spinaveraged case (upper plots) and the spin difference (lower plots). The next important contribution is the $\eta$-decay $\eta \rightarrow 2 \gamma$ with $10 \%-20 \%$.

Comparing figure 9 and figure 10 one realizes that the rate of the QCD-Compton process is several times larger than that of the $g g$-scattering process in the spin averaged case (upper plots). For the spin-difference they are comparable at low $p_{\perp}$ even with the parametrisation of Altarelli\&Stirling with its large gluon polarisation. Due to the much faster decrease with $p_{\perp}$ of the latter the QCD-Compton process becomes also in the polarised case the dominant one at high- $p_{\perp}$. This dominance will be more pronounced for parametrisations with a smaller $\Delta g$.

From the discussion above it follows that the mesons from which those background photons stem have to be reconstructed, in order that they can be separated from the true signal. There are two main possibilities that the mesons escape their reconstruction and thus 'fake' photons remain. The two possible sources of 'fake' $\gamma$ 's are the following. First, asymmetric decay, i. e. one $\gamma$ is inside the detector, the other outside, such that they cannot be combined. Second, merged $\gamma$ 's, i. e. the two $\gamma$ 's are too narrow and cannot be resolved by the detector. Investigations have been done only for the latter case, because this is more general, whereas the former is dependent on the precise detector geometry and requires a specialized detector simulation.

In the following we determine the rate of 'fake' $\gamma$ 's from pion decay in dependence of the spatial detector resolution. The minimal opening 
angle of a $\gamma$-pair in the rest frame of the pion is given by:

$$
\chi_{\min }=2 \frac{m_{\pi}}{E_{\pi}} .
$$

The following resolutions of the detector are considered

$$
\begin{aligned}
& \chi^{\text {res }}>0,005 \mathrm{rad} \\
& \chi^{\text {res }}>0,01 \mathrm{rad} \\
& \chi^{\text {res }}>0,02 \mathrm{rad},
\end{aligned}
$$

For the PHENIX detector the planned design would result in $\chi^{\text {res }}>$ $0,01 \mathrm{rad}$. We define the fake- $\gamma$-rate $R$ as the fraction of the number of unresolved pions and the total number of pions:

$$
R=\frac{N_{\pi}^{\text {unres }}}{N_{\pi}}
$$

The $\mathrm{MC}$ fake- $\gamma$-rate $R_{\mathrm{MC}}$ has to be transformed to the experimental $R_{\exp }$ as follows:

$$
R_{\exp }^{\uparrow \downarrow(\uparrow \uparrow)}=\frac{R_{\mathrm{MC}}^{\uparrow \downarrow(\uparrow \uparrow)}+\rho R_{\mathrm{MC}}^{\uparrow \uparrow(\uparrow \downarrow)}}{1+\rho}
$$

with

$$
\rho \equiv \frac{1-P}{1+P} \times \frac{1-A_{\mathrm{MC}}}{1+A_{\mathrm{MC}}} .
$$

Then the number of real direct photons is given by:

$$
N_{\gamma}=N_{\text {tot }}-R N_{\pi} .
$$

The total number of fake- $\gamma$ 's in dependence of their opening angle and the fake- $\gamma$-rates for the different resolutions considered of the detector are displayed in figure 11. These plots show that the PHENIX resolution of $\chi^{\text {res }}>0,01$ is sufficient to keep the fake- $\gamma$-rate below $10 \%$ up to a transverse momentum of $p_{\perp} \approx 20 \mathrm{GeV}$. Due to the steep slope of the $\gamma$-rates around $\chi=0,01$ in the upper plots the resolution $\chi^{\text {res }}>0,02$ is absolutely unsuited, whereas the better resolution $\chi^{\text {res }}>0,005$ is excellent. Further studies are needed to determine the optimal compromise between costs and fake- $\gamma$-rates for the detector. In addition there is a slight spin dependence of the fake- $\gamma$-rates $R^{\uparrow \uparrow}<R^{\uparrow \downarrow}$ such that unless $\chi^{\text {res }}=0,005$ a carefull simulation is needed to determine the resulting corrections and thus keep the systematic uncertainties small. 


\section{Prompt- $\gamma$ and Away-Side Jet}

Additional information can be extracted from prompt- $\gamma$ measurements if they are observed in coincidence with jets, which could also help to reduce unwanted background. The resulting information could e.g. be used to determine the $x$-dependence of the gluon polarisation $\Delta g(x)$ which is not sufficiently determined by the prompt- $\gamma$ signal alone. In practice, however, one will determine all the polarised distribution functions by simultaneous fits to all data. In the context of such fits prompt- $\gamma$ 's plus jet data could he of great importance for $\Delta g\left(x, Q^{2}\right)$. Let us describe first how we handled the jet-reconstruction. Our Monte-Carlo code just like PYTHIA uses the jet routines of JETSET. Jet reconstruction was thus done by the JETSET-subroutine LUCELL [22]. This routine is also used to analyse unpolarised proton-proton collisions and defines jets in the two-dimensional $(\eta-\phi)$ - plane, $\eta$ being the rapidity and $\phi$ the angle around the $z$-axes. For our calculations we used $25 \eta$-bins and $24 \phi$-bins with various bounds for $|\eta|$. The jet defining algorithm works as follows. First all transverse energies $E_{\perp}=\sqrt{p_{\perp}^{2}+m^{2}}$ in a bin are summed. If this sum exceeds a certain value $\left(E_{\perp}^{\text {cell }}\right)$, which we chose to be $1.5 \mathrm{GeV}$, than it is treated as a jet candidate. Starting from the cell with the highest transverse energy all cells in a smaller 'distance' than $R=\sqrt{(\Delta \eta)^{2}+(\Delta \phi)^{2}}$ (which we chose as 0.7) are combined to a 'cluster' and if the total transverse energy in this cluster exceeds $E_{\top}^{\text {cluster }}$ which we chose to be $3.5 \mathrm{GeV}$, the contained particles are accepted as a jet.

Finally we use an additional constraint on $|\eta|$ implied by the present design of the STAR detector. This detector is planned to be built with so-called end-caps, giving them a wider $\eta$-range. With these end-caps

it covers the range $\left|\eta^{\gamma}\right| \leq 1,\left|\eta^{\text {Jet }}\right| \leq 0.3$ while without them only the range $\left|\eta^{\gamma}\right| \leq 2,\left|\eta^{\mathrm{Jet}}\right| \leq 1.3$. is accessible.

We start by showing that the larger $\eta$ range is really needed to do allow for a sensible jet analyses. Figure 12 shows a rapidity distribution of the jets generated by our code and the consequences of the rapidity cuts. In each case the area of one of the small rectangels measures the logarithm of the number of events. Obviously the rapidity cuts without end-caps are too restrictive while adding the end-caps allows to cover nearly all of the interesting rapidity-range. 
We are interested in the Compton-process of figure 1. Thus we require that the jet and the photon are detected with a relative angle above 90 degrees in the partonic center of momentum system. In the following, jets fullfilling this criterium are called away-side jets. In the hadronic center of momentum system, which for RHIC coincides with the laboratory system this condition looks rather complicated due to the Lorentz-boost

$$
\cos \chi=\frac{4 x_{1} x_{2} \cos \left(\phi^{\gamma}-\phi^{\mathrm{Jet}}\right)+\left(x_{2} e^{\eta^{\gamma}}-e^{-\eta^{\gamma}}\right)\left(x_{2} e^{\eta^{\mathrm{Jet}}}-e^{-\eta^{\mathrm{Jet}}}\right)}{\left(x_{2} e^{\eta^{\gamma}}+e^{-\eta^{\gamma}}\right)\left(x_{2} e^{\eta^{\mathrm{Jet}}}+e^{-\eta^{\mathrm{Jet}}}\right)}<0
$$

but it turned out that for the jets we generated this criterium is actually equivalent to the much simpler one

$$
\cos \left(\phi^{\gamma}-\phi^{\mathrm{Jet}}\right)<0 \quad .
$$

The consequences of this criterium are shown in figure 13. In this figure we show histograms of the generated jets as a function of the photon and jet rapidity. If the 'away-side-jet' criterium is not used we get the results on the left side for the spin averaged and spin difference rates. Obviously the photons are in general very strongly correlated with the jet axes. If the criterium is applied to single out the hard Compton processes the distributions on the right side are obtained. Obviously these are only a small fraction of the total events and the rapidities are substantially different. The weak remaining correlation is a consequence of the Lorentz-boost. In the partonic center of momentum system the rapidities are anti-correlated.

The cross sections for photon-plus-jet events are related to the distribution functions according to

$$
\begin{aligned}
\frac{d^{3} \sigma(p p \rightarrow \gamma+\mathrm{Jet}+X)}{d p_{\perp}^{2} d \eta^{\gamma} d \eta^{\mathrm{Jet}}} & \sim \sum_{q} e_{q}^{2} q\left(x_{a}\right) g\left(x_{b}\right) \frac{d \sigma(q g \rightarrow \gamma q)}{d \hat{t}} \\
\frac{d^{3} \Delta \sigma(p p \rightarrow \gamma+\mathrm{Jet}+X)}{d p_{\perp}^{2} d \eta^{\gamma} d \eta^{\mathrm{Jet}}} & \sim \sum_{q} e_{q}^{2} \Delta q\left(x_{a}\right) \Delta g\left(x_{b}\right) \frac{d \Delta \sigma(q g \rightarrow \gamma q)}{d \hat{t}}
\end{aligned}
$$

From this we can calculate the differential $\gamma$-jet cross sections as a function of $\eta^{\gamma}$ and $\eta^{\text {Jet }}$. Figure 14 and 15 show the results for spin average and spin difference in the $\eta^{\gamma}=0, \eta^{\mathrm{Jet}}=0$ bin for our two parametrisations with large $\Delta g$.

Photon-jet experiments give actually more information than contained in figure 14 and 15 . It is possible to reconstruct the $x$-values 
from the measured pseudorapidities and transverse momenta. In the ideal case, neglecting all initial and final state interaction this connection is simply given by

$$
\begin{aligned}
p_{1} & =x_{1}(P, 0,0, P) \\
p_{2} & =x_{2}(P, 0,0,-P) \\
p^{\gamma} & =p_{\perp}\left(\cosh \eta^{\gamma}, \cos \phi^{\gamma}, \sin \phi^{\gamma}, \sinh \eta^{\gamma}\right) \\
p^{\mathrm{Jet}} & =p_{\perp}\left(\cosh \eta^{\mathrm{Jet}}, \cos \phi^{\mathrm{Jet}}, \sin \phi^{\mathrm{Jet}}, \sinh \eta^{\mathrm{Jet}}\right) .
\end{aligned}
$$

where $p_{1}$ and $p_{2}$ are the four-momenta of the incomming partons. Energy-momentum conservation implies than (neglecting all masses)

$$
\begin{aligned}
& x_{1} \simeq \frac{2 p_{\perp}}{\sqrt{s}}\left(\frac{e^{\eta^{\gamma}}+e^{\eta^{\mathrm{Jet}}}}{2}\right) \\
& x_{2} \simeq \frac{2 p_{\perp}}{\sqrt{s}}\left(\frac{e^{-\eta^{\gamma}}+e^{-\eta^{\mathrm{Jet}}}}{2}\right) .
\end{aligned}
$$

Finally to decide which of these x-values belongs to the gluon and which to the quark the following procedure was suggested: Define $x_{a}=\min \left(x_{1}, x_{2}\right)$ and $x_{b}=\max \left(x_{1}, x_{2}\right)$ and require $x_{b} \geq 0.2$. As the gluon distribution is already small at such $x$-values one can expect that $x_{b}$ is the quark momentum fraction.

In proceeding like this a number of rather severe asumptions were made such that it was rather unclear how good it would work. SPHINX gives us the possibility to check it explicitely. Figure 16 to 19 show the results. Figure 16 shows just histograms of the generated quark and gluon momentum fractions. Figure 17 shows how these are correlated with $x_{a}, x_{b}, x_{g}^{\exp }, x_{q}^{\exp }$. Again the area of the rectangles is proportional to the logarithm of the rate, such that the correlations are much stronger than they look. The figures on the left side show how the $x$ values of the distribution functions $x^{\mathrm{MC}}$ are correlated to those generated by the complete SPHINX algorithm, i.e. the difference between $x_{g}^{\exp }$ and $x_{g}$ respectively $x_{q}^{\exp }$ and $x_{q}$ is entirely due to the intrinsic transverse momentum and to effects of the initial and final state showering. The identification of the jets is taken from the Monte-Carlo, such that there are no misidentifications. In an ideal experiment $x_{g}^{\exp }$ and $x_{q}^{\exp }$ are the best measurable approximations for $x_{g}$ and $x_{q}$. The right side shows how good the simple procedure just described is able to reconstruct the $x$-values, still without the $x_{b}$-cut. 
Obviously the quark momentum fraction can be reconstructed quite reasonably, while the reconstruction of $x_{g}$ is problematic. This is why a $x_{b}$ cut is needed. Introducing it the correlation becomes much better, as shown in more detail in figure 18 and 19 for $g(x)$ and $\Delta g(x)$. In these figures the upper graphs shows the histogram of the $x$ values actually chosen by the Monte Carlo code. The lower ones show the distribution of $x$-values reconstructed with the described procedure. The agreement is very good for $x>0.04$ and gets rapidly bad if one goes to smaller $x$-values. The problems are far less pronounced for the polarised case because the chosen function for $\Delta g(x)$ is comparably small at small $x$. For a situation where $\Delta g(x)$ would be concentrated at extremely small $x$ it could not be deduced from the $\gamma$-jet signal. The latter is however true for all polarised experiments. Such a very soft polarised gluon distribution would also not be detected in deep inelastic lepton-nucleon scattering such that it could not help to explain the observed data. Its only effect could be to screw up the extrapolation to small- $x$ needed to derive experimental values for the sum rules. If the observed data are interpreted as giving evidence for an anomalous gluon contribution (however one is trying to define it) than $\Delta g(x)$ cannot be too soft and thus should be seen by RHIC.

With all these caveats one should note, however, that our simulation shows, that an actual prompt- $\gamma$-plus-jet experiment would be able to distinguish even between the two rather similar gluon distributions we used (see figure 19, the bottom plots).

We conclude this paragraph by stating that prompt- $\gamma$-jet coincidences will give interesting data but that their analyses will be highly non-trivial requirering extensive numerical simulations. A cut like $x_{b} \geq 0,2$ is necessary. While a more restrictive cut leads to a better reconstruction it also worsens statistic. We hope that SPHINX will help to find the best compromise.

\section{$4 \quad \pi^{0}$-Production}

We discussed already in section 2 that the $\pi^{0}$ decaying into two photons have to be reconstructed in order to extract the direct- $\gamma$ signal. This should be possible with high efficency. However, this opens also the possibility to use the $\pi^{0}$ asymmetry as an independent measurement of the gluon polarisation as well. Their advantage in comparison 
with the prompt- $\gamma$ 's is the larger cross section and hence the smaller statistical error. On the other hand the pions are a less direct probe for $\Delta g$ because several processes contribute to their rate, such that the total observed asymmetry depends in a rather involved manner on the gluon polarisation. For example the most important contribution, the QCD-Compton process is proportional to $\Delta g$, but the next important one $q q \rightarrow q q$ is independent of $\Delta g$, whereas in $g g \rightarrow g g$ it enters twice. Again a detailed Monte-Carlo simulation should allow to relate the data to the distribution functions.

In figure 20 the contributions of the QCD-Compton process and the $g g$-scattering process to the $\pi^{0}$-production are displayed. In the spin averaged case the QCD-Compton process is the far more important. Due to the large gluon polarisation in Altarelli\&Stirling, which enters in the $g g$-scattering process twice, this process gives the major contribution at low $p_{\perp}$. However, because it decreases much faster with $p_{\perp}$, for $p_{\perp}>8 \mathrm{GeV}$ the QCD-Compton process becomes the dominant one in the polarised case too, even for large gluon polarisation. Nevertheless, the $g g$-scattering process leads to an asymmetry much larger than the QCD-Compton process. Here, the MC-statistic gives reliable predictions only up to $p_{\perp}<15 \mathrm{GeV}$. The different $p_{\perp^{-}}$ dependence of the individual processes could be used to disentangle them.

Figure 21 shows the yield of pions due to the QCD-Compton process in the spin averaged case (upper plot), for the spin-difference (middle) and the resulting asymmetry (lower plot) as a function of $p_{\perp}$ for parametrisations with a large gluon polarisation. While figure 20 showed results for $x_{F}=0$ figure 21 shows the rates integrated over $x_{F}$. In comparison with the prompt- $\gamma$-datas the two parametrisation of Altarelli\&Stirling (squares) and Ross\&Roberts set D (triangles) are much harder to distinguish on the base of these rates. Although this is unfortunate in this case it shows that one is really analysing an observable which tests different properties than the prompt- $\gamma$-measurements.

\section{Summary}

We have analysed three observables, namely direct prompt- $\gamma$ 's, prompt-

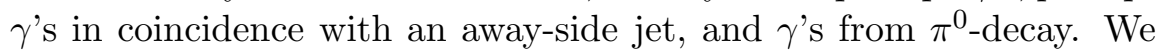
found that each of them should allow to obtain significant results in 
RHIC spin-physics experiments. These results should allow to settle the problem of $\Delta g(x)$. However, each of these signals has its problems: For direct photons the background rate in rather high, for prompt- $\gamma$ jet coincidences the jet reconstruction is non-trivial and has, just as for the $\pi^{0}$-decay, to rely very heavily on Monte-Carlo simulations. Consequently the existence of several independent codes will be crucial to relate the observed data in a reliable way to the basic physical quantities of interest. We hope that our code will contribute to this endeavour.

\section{Acknowledgement}

We are very much indepted to T. Sjöstrand for his help in understanding the details of PYTHIA and for his encouragement. A.S. thank DFG, BMFT, and the MPI Heidelberg for support. L.M. has been supported by KBN grant 2-P302-143-06. 


\section{References}

[1] EMC, J. Ashman et al., Phys. Lett. B206 (1988) 364

EMC, J. Ashman et al., Nucl. Phys. B328 (1990) 1

[2] SMC, B. Adeva et al., Phys. Lett. B302 (1993) 533

SMC, D. Adama et al.,Phys. Lett. B239 (1994) 399

[3] P.L. Anthony et al., Phys. Rev. Lett. 71 (1993) 959

[4] S.A. Larin and J.A.M. Vermaseren, Phys. Lett. B 259 (1991) 345

I.I. Balitsky, V.M. Braun and A.V. Kolesnichenko, Phys. Lett. B 242 (1990) 245; B318 (1993) 648(E).

E. Stein et al., 'QCD Sum Rule Calculation of Twist-3 Contribution to Polarised Nucleon Structure Functions', UFTP preprint 366/1994, HEP-PH-9409212

X. Ji and P. Unrau, Phys. Lett. B333 (1994) 228

B. Ehrsperger, L. Mankiewicz, and A. Schäfer, Phys. Lett. B323 (1994) 439

J. Ellis and M. Karliner, Phys. Lett. B313 (1993) 131

F.E. Close and R.G. Roberts, Phys. Lett. B316 (1993) 165

G. Altarelli, P. Nason, and G. Ridolfi, Phys. Lett. B320 (1994) 152

[5] B. Ehrnsperger et al., Phys. Lett. B321 (1994) 121

[6] C.S. Lam and B.-A. Li, Phys. Rev. D25 (1982) 683

R.D. Carlitz, J.C. Collins, and A.H. Mueller, Phys. Lett. B214 (1988) 229

G. Altarelli and G.G. Ross, Phys. Lett. B212 (1988) 3911

G. Bodwin and J. Qiu in [7]

A.V. Manohar in [7]

J. Ellis, M. Karliner, and C.T. Sachrajda, Phys. Lett. B231 (1989) 497

U. Ellwanger, Phys. Lett. B259 (1991) 469

L. Mankiewicz and A. Schäfer, Phys. Lett. B242 (1990) 455 R.L.

Jaffe and A. Manohar, Nucl. Phys. B337 (1990) 509

[7] Proceedings of the 'Polarized Collider Workshop', University Park, PA 1990,

edts. J. Collins, S.F. Heppelman, and R.W. Robinett, AIP conf. proc. No 223, New York 1991

[8] St. Güllenstern et al., Phys. Lett. B312 (1993) 166 
[9] G. Bunce et al., Particle World 3 (1992) 1

Joined PHENIX-STAR spin proposal 1993

[10] E.L. Berger and J. Qiu, Phys. Rev. D40 (1989) 778, Phys. Rev. D40 (1989) 3128, and J. Qiu in [7]

C. Bourelly, J. Ph. Gullet, and J. Soffer, Nucl Phys. B 361 (1991) 72

[11] T. Sjöstrand, CERN-TH.6488/92; T. Sjöstrand, Comp. Phys. Comm. 39 (1986) 347; T. Sjöstrand and M. Bengtsson, Comp. Phys. Comm. 43 (1987) 367.

[12] S. Güllenstern et al., preprint, submitted to Comp. Phys. Comm.

[13] L.E. Gordon and W. Vogelsang, Phys. Rev. D48 (1993) 3136

A.P. Contagouris et al., Phys. Lett. B 304 (1993) 329

[14] M. Karliner and R.W. Robinett, Phys. Lett. B324 (1994) 209

[15] The HERMES Technical Design Report, DESY-PRC 93/06, MPIH-V20-1993

[16] G. Altarelli and J. Stirling, Particle World 1 (1989) 40.

[17] G. G. Ross and R. G. Roberts, Rutherford preprint RAL-90-062 (1990).

[18] G. Altarelli and G. Parisi, Nucl. Phys. B175 (1977) 298

[19] R. Gastmans and Tai Tsun Wu, The Ubiquitous Photon - Helicity Method for $Q E D$ and $Q C D$, Oxford Science Publications (1990); K. Hidaka Nucl. Phys. B192 (1981) 369

[20] H.-U. Bengtsson and T. Sjöstrand, Computer Phys. Commun. 46 (1987) 43.

[21] M. Glück, E. Reya, and A. Vogt, Z. Phys. C48 (1990) 471

[22] T. Sjöstrand, PYTHIA 5.6 and JETSET 7.3, Physics and Manual, CERN-TH.6488/92 May 1992 (revised Sept. 1992) 


\begin{tabular}{|lrr|}
\hline Subprocess & $|\bar{M}|^{2}$ & $|\Delta \bar{M}|^{2}$ \\
\hline$q g \rightarrow \gamma q$ & $-e_{q}^{2} \frac{1}{3}\left(\hat{s}^{2}+\hat{t}^{2}\right) /(\hat{s} \hat{t})$ & $-e_{q}^{2} \frac{1}{3}\left(\hat{s}^{2}-\hat{t}^{2}\right) /(\hat{s} \hat{t})$ \\
\hline$q \bar{q} \rightarrow \gamma g$ & $e_{q}^{2} \frac{8}{9}\left(\hat{t}^{2}+\hat{u}^{2}\right) /(\hat{t} \hat{u})$ & $-e_{q}^{2} \frac{8}{9}\left(\hat{t}^{2}+\hat{u}^{2}\right) /(\hat{t} \hat{u})$ \\
\hline
\end{tabular}

Table 1: The squarred matrix elements for the Comptonand annihilation process 


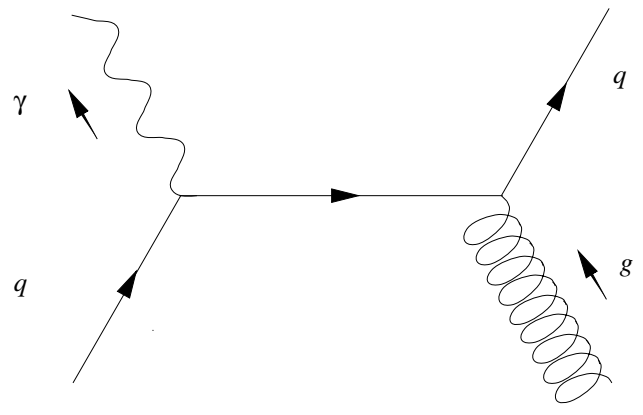

Figure 1: The Compton graph 


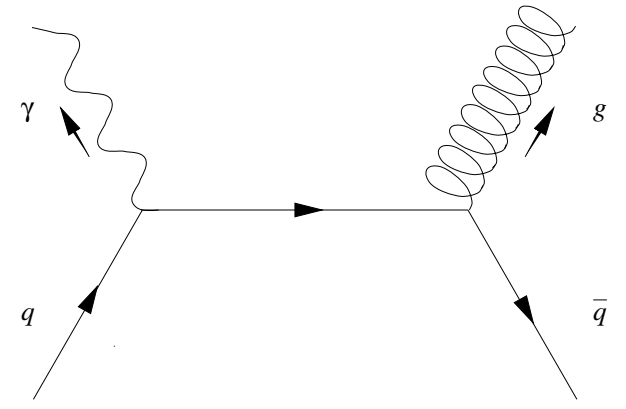

Figure 2: The annihilation graph 


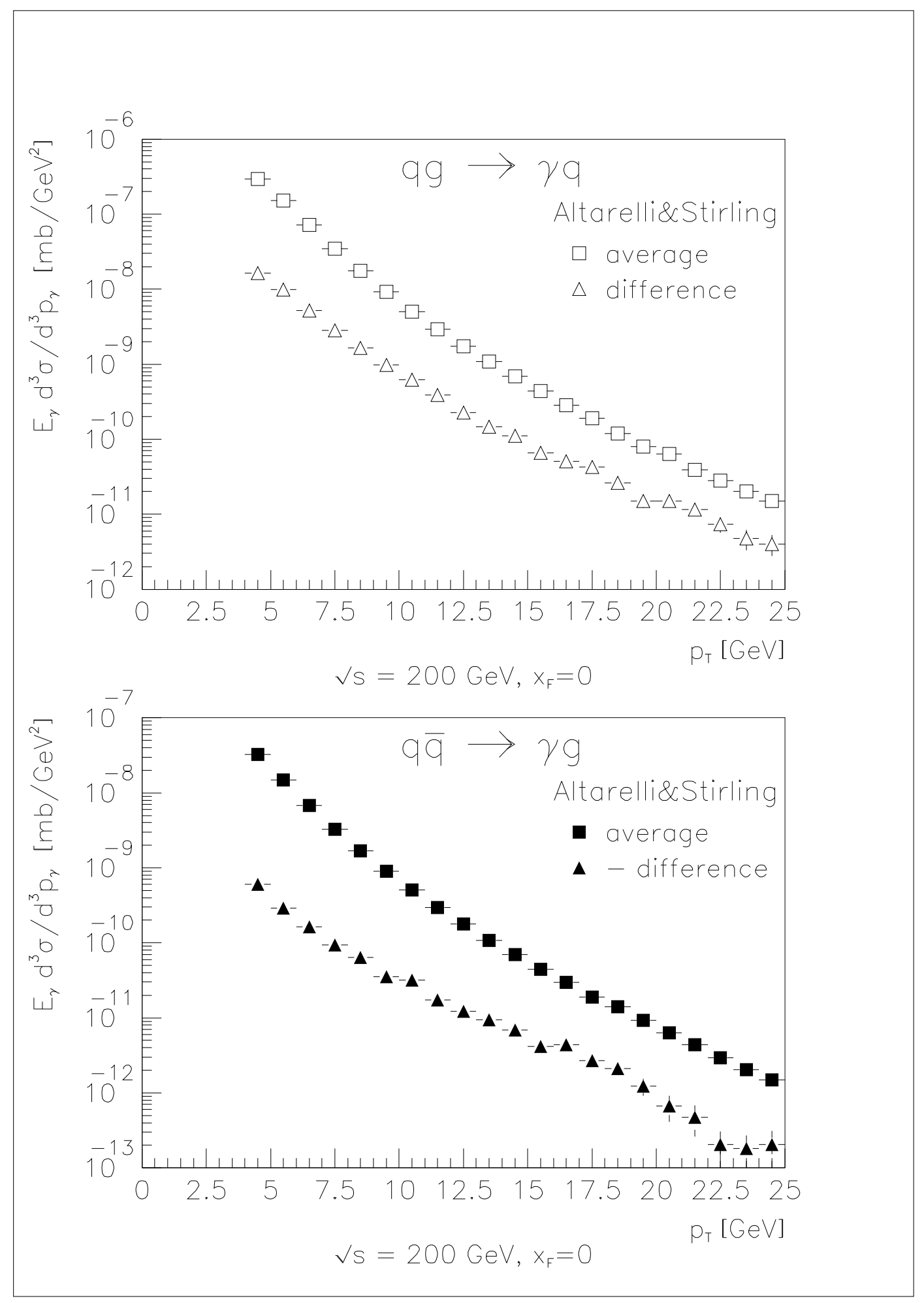

Figure 3: Spin-average and spin-difference cross sections for prompt $\gamma$ production. (Note that in the lower plot the negative difference was plotted.)

top: The Compton process $\quad$ bottom: The annihilation process 


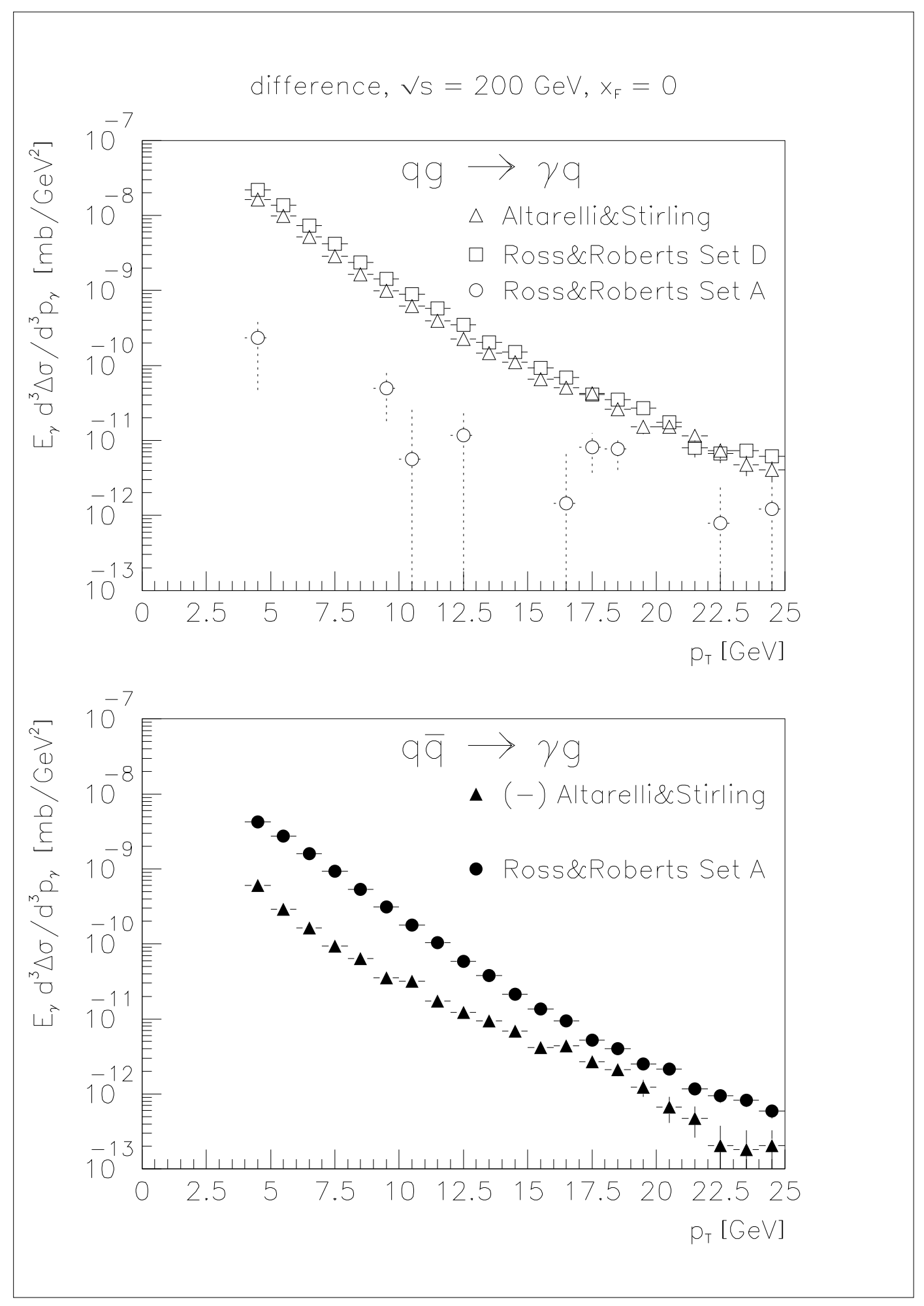

Figure 4: Spin-difference cross sections for prompt- $\gamma$ production for various parametrisations. (Note that in the lower plot the negative difference was plotted for the Altarelli\&Stirling parametrisation.)

top: The Compton process bottom: The annihilation process 


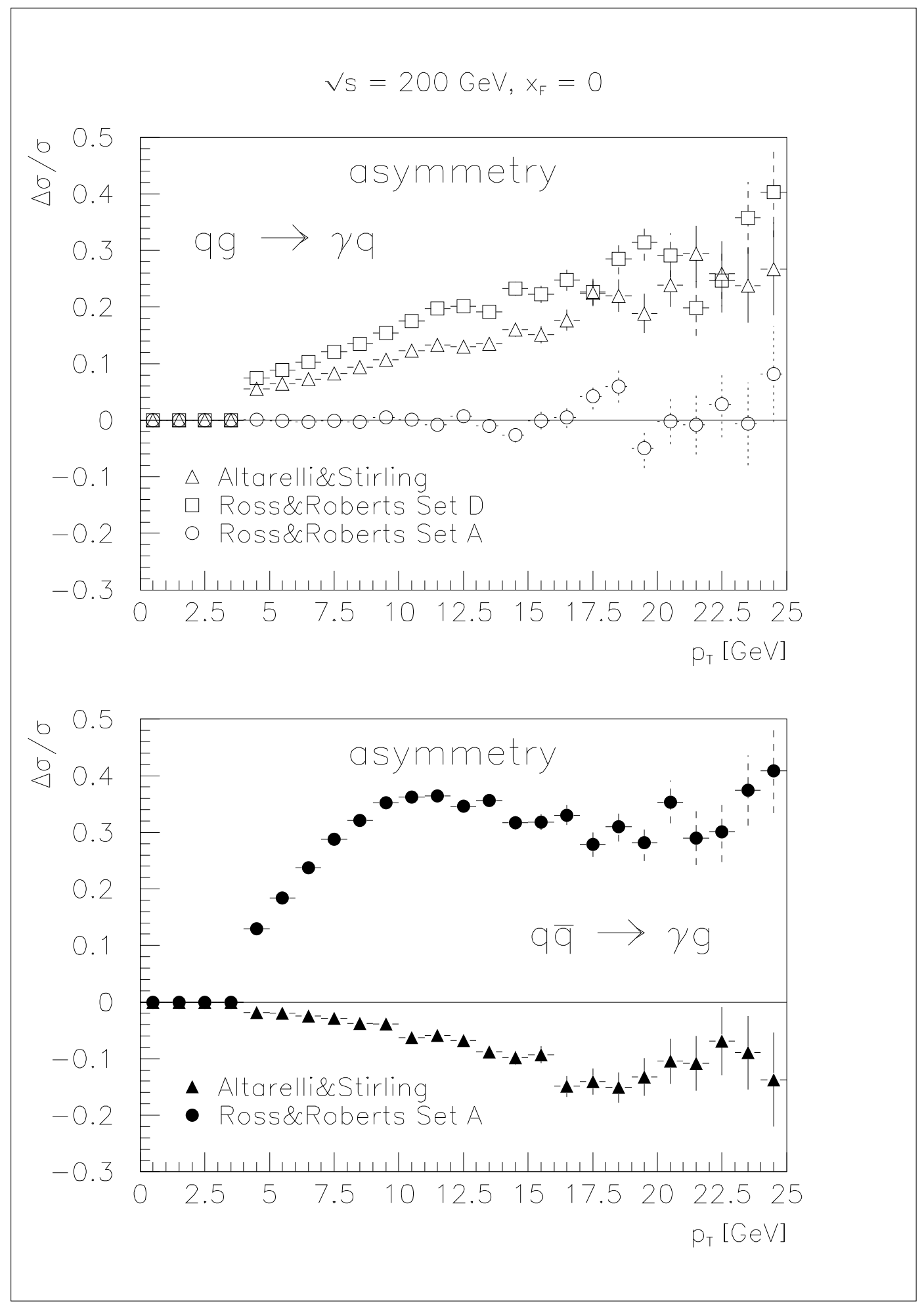

Figure 5: The asymmetry for prompt- $\gamma$ production for various parametrisations

top: The Compton process bottom: The annihilation process 


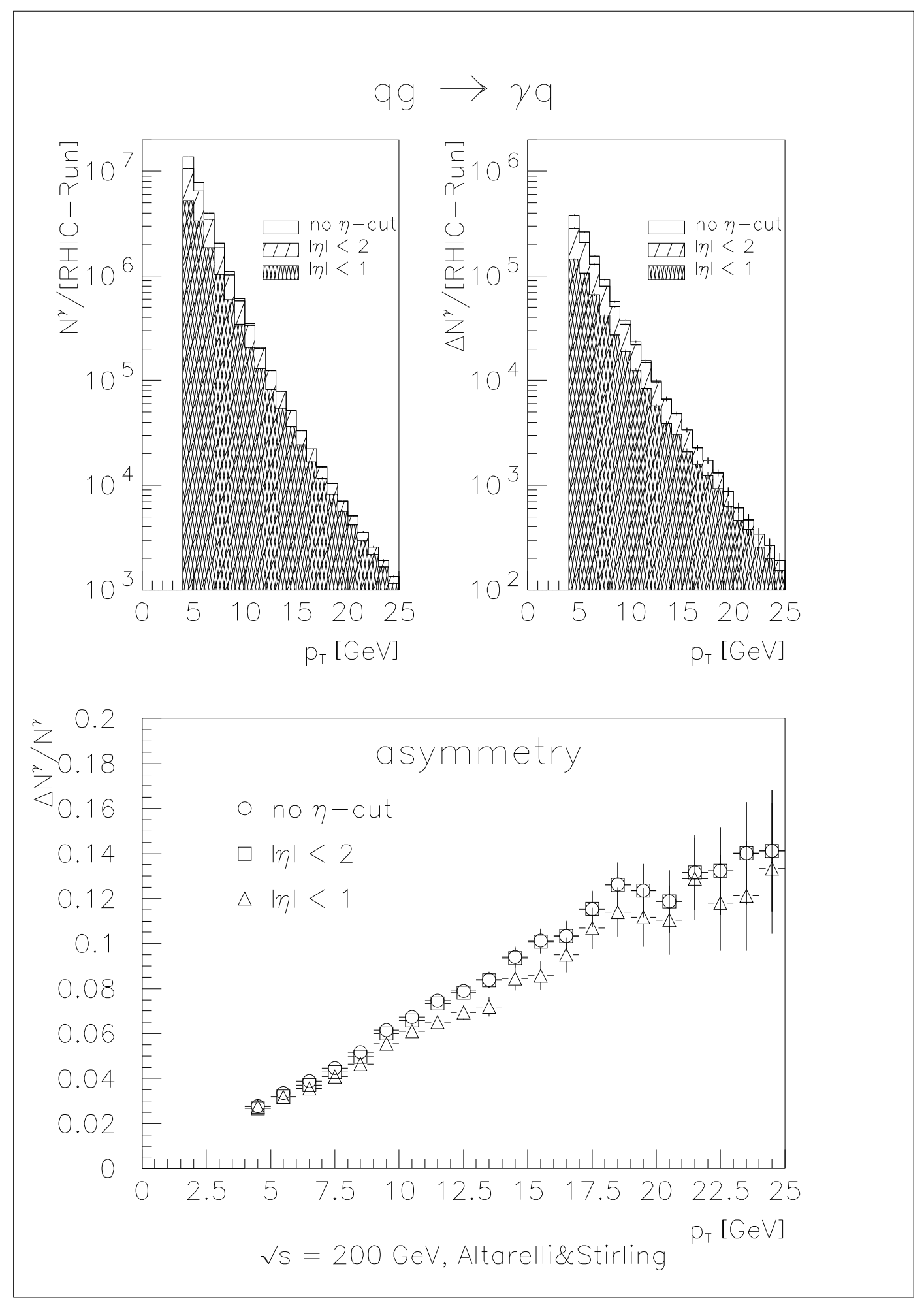

Figure 6: Influence of the $\eta$-cut on prompt- $\gamma$ production top left: spin average top right: spin difference bottom: asymmetry 


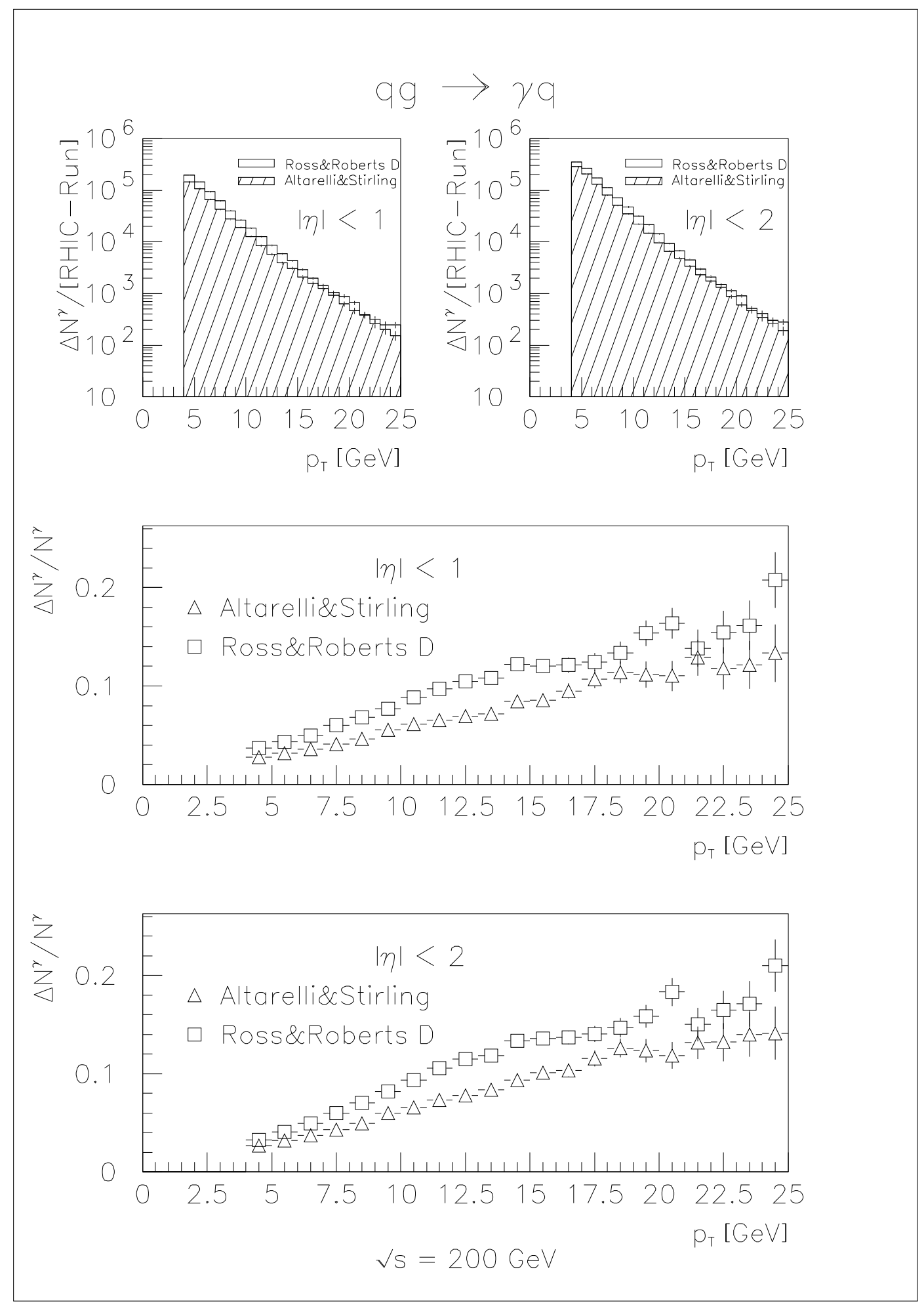

Figure 7: Consequences of the $\eta$-cut for two different parametrisations

top left: spin difference, $|\eta|<1$ middle: asymmetry, $|\eta|<1$ top right: spin difference, $|\eta|<2$

bottom: asymmetry, $|\eta|<2$ 


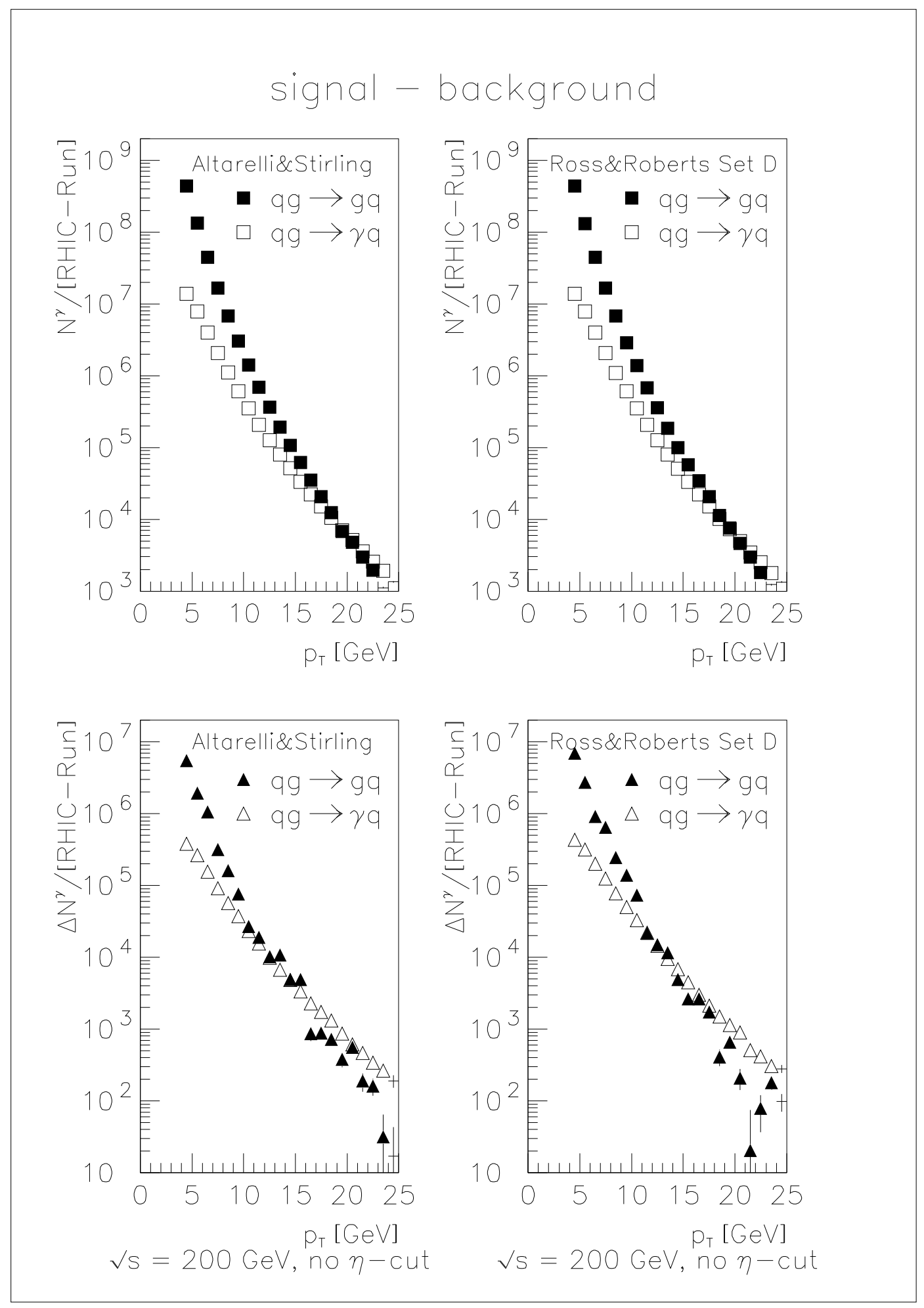

Figure 8: Comparison of signal and background top: spin average left: Altarelli\&Stirling bottom: spin difference

right: Ross\&Roberts set $D$ 


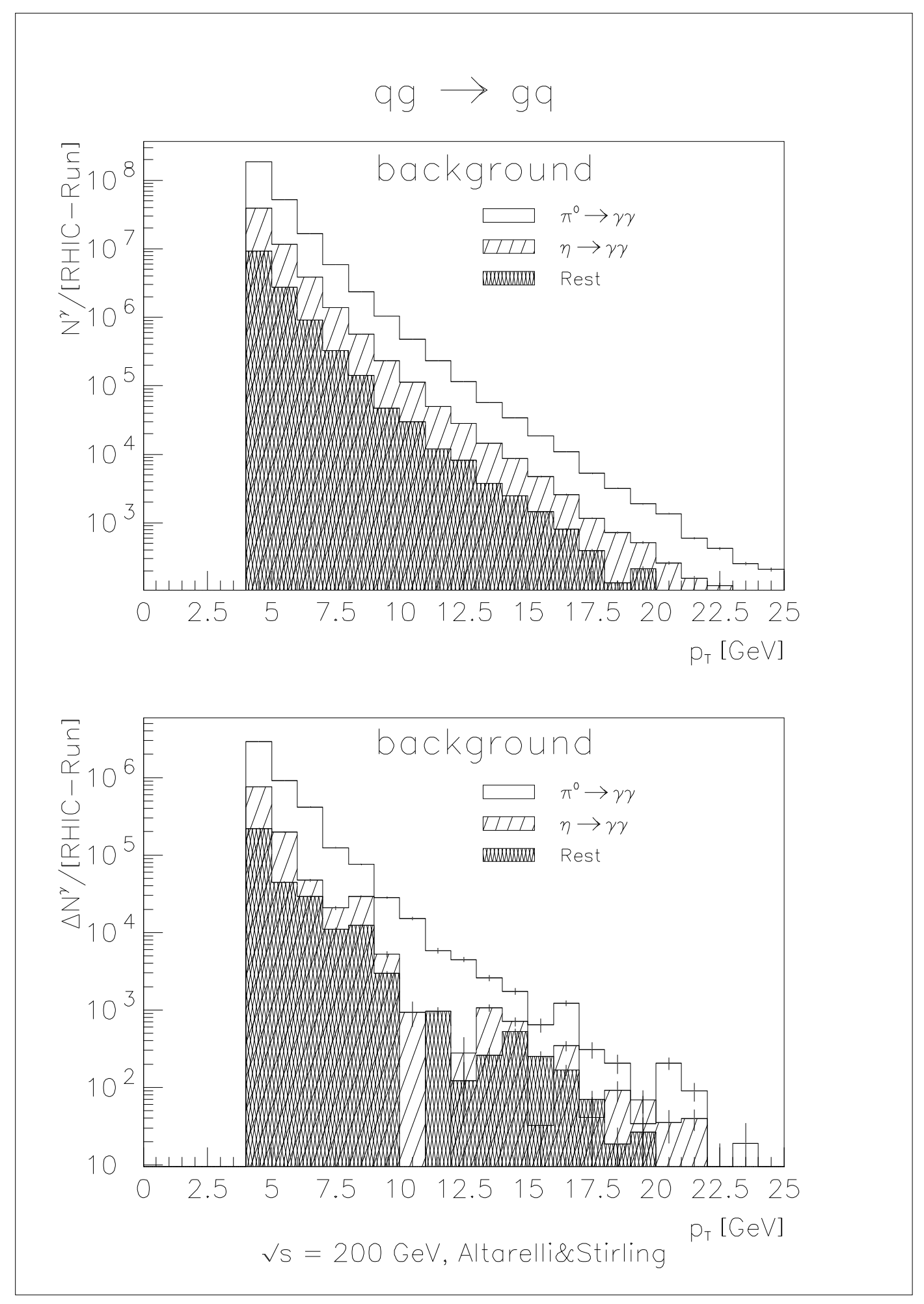

Figure 9: Composition of the background for $q g \rightarrow g q$ top: spin average bottom: spin difference 


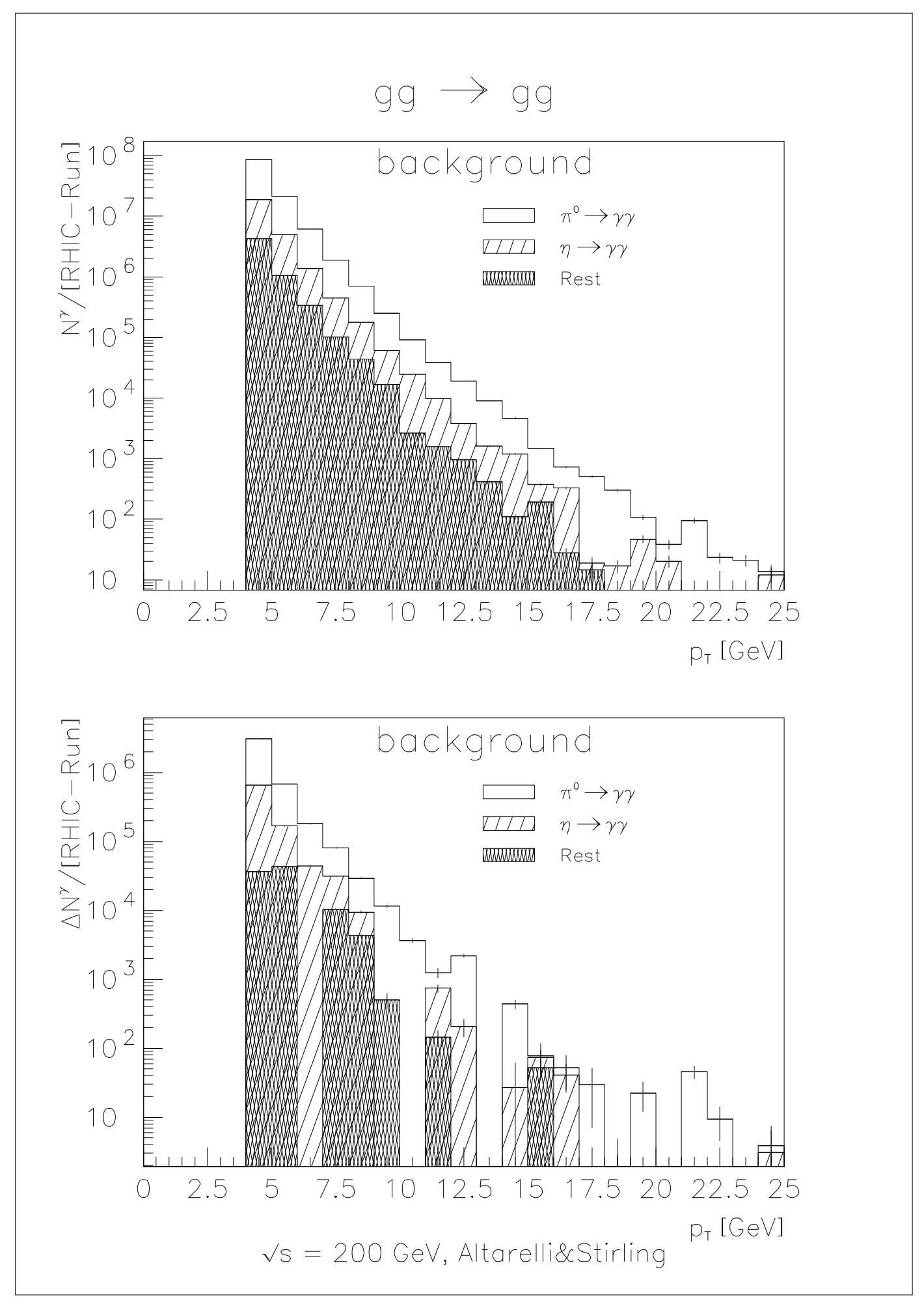

Figure 10: Composition of the background for $g g \rightarrow g g$ top: spin average bottom: spin difference 

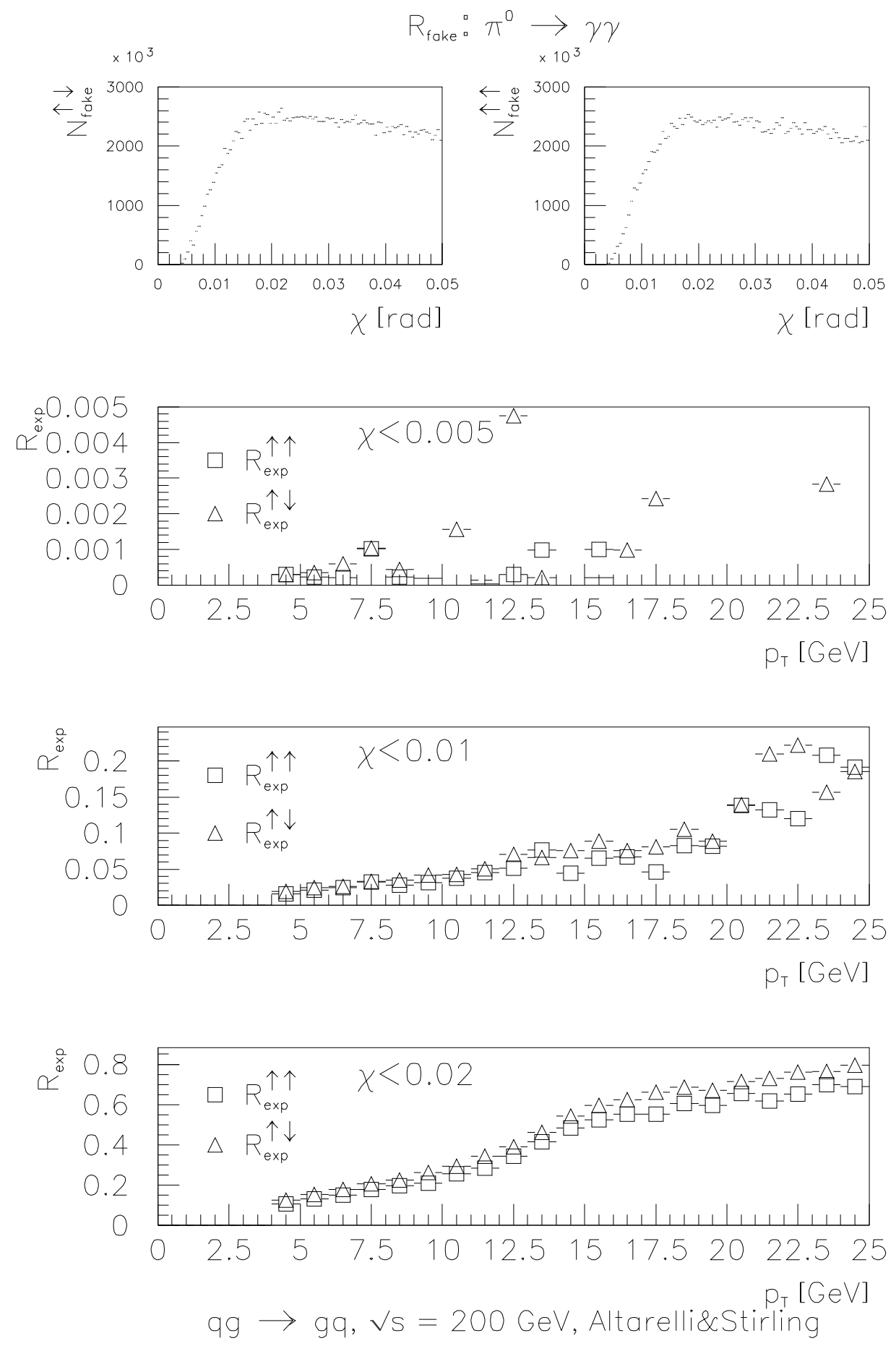

Figure 11: Fake- $\gamma$-rates

1. line: distribution of opening angles for the photon pairs

2.-4. line: fake- $\gamma$-rates for different resolutions 


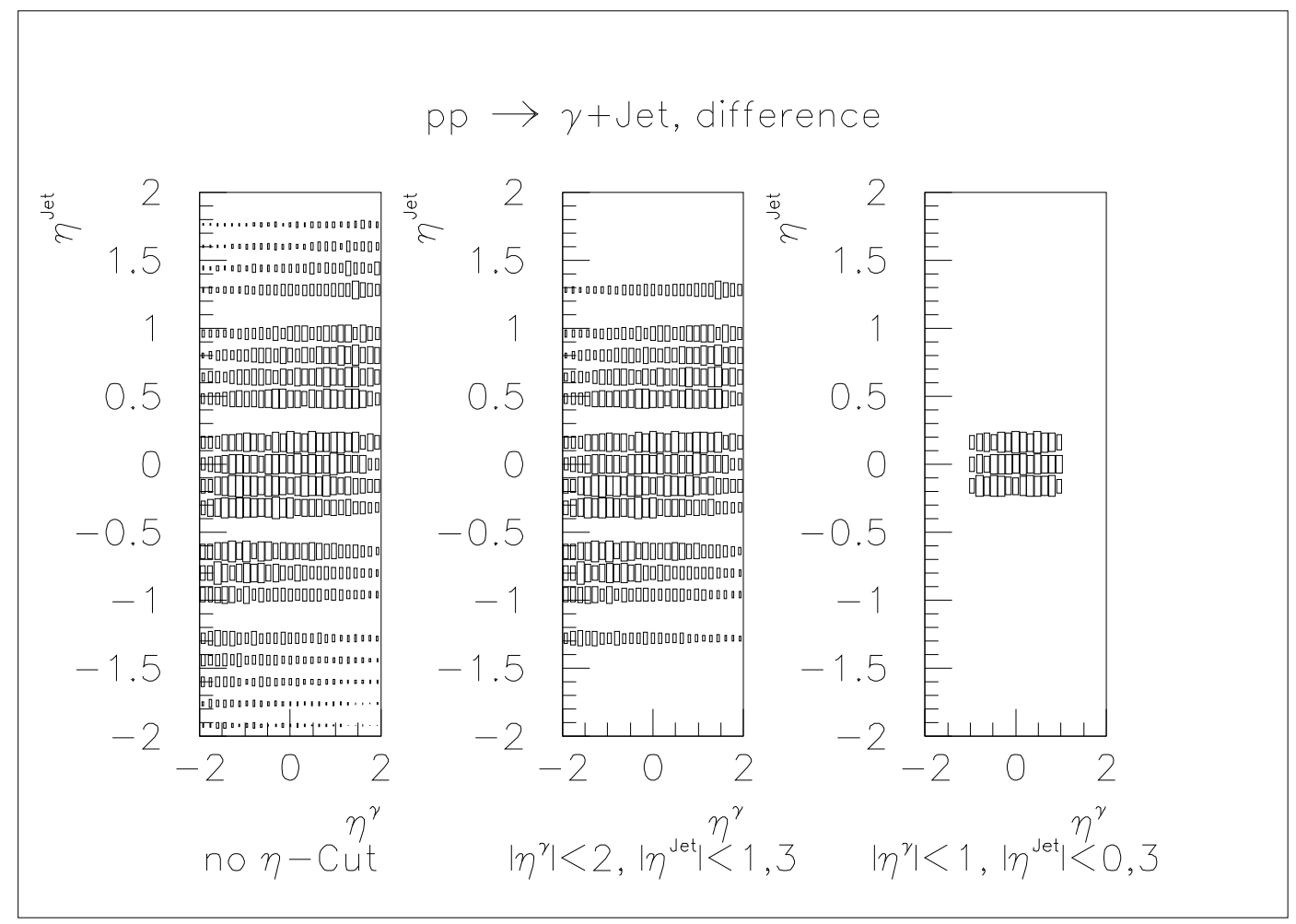

Figure 12: $\gamma+$ jet-production for different $\eta$ cuts. The areas of the rectangles are proportional to the logarithm of the counts.

left: no $\eta$ cut

middle: STAR with end caps

right: STAR without end caps 


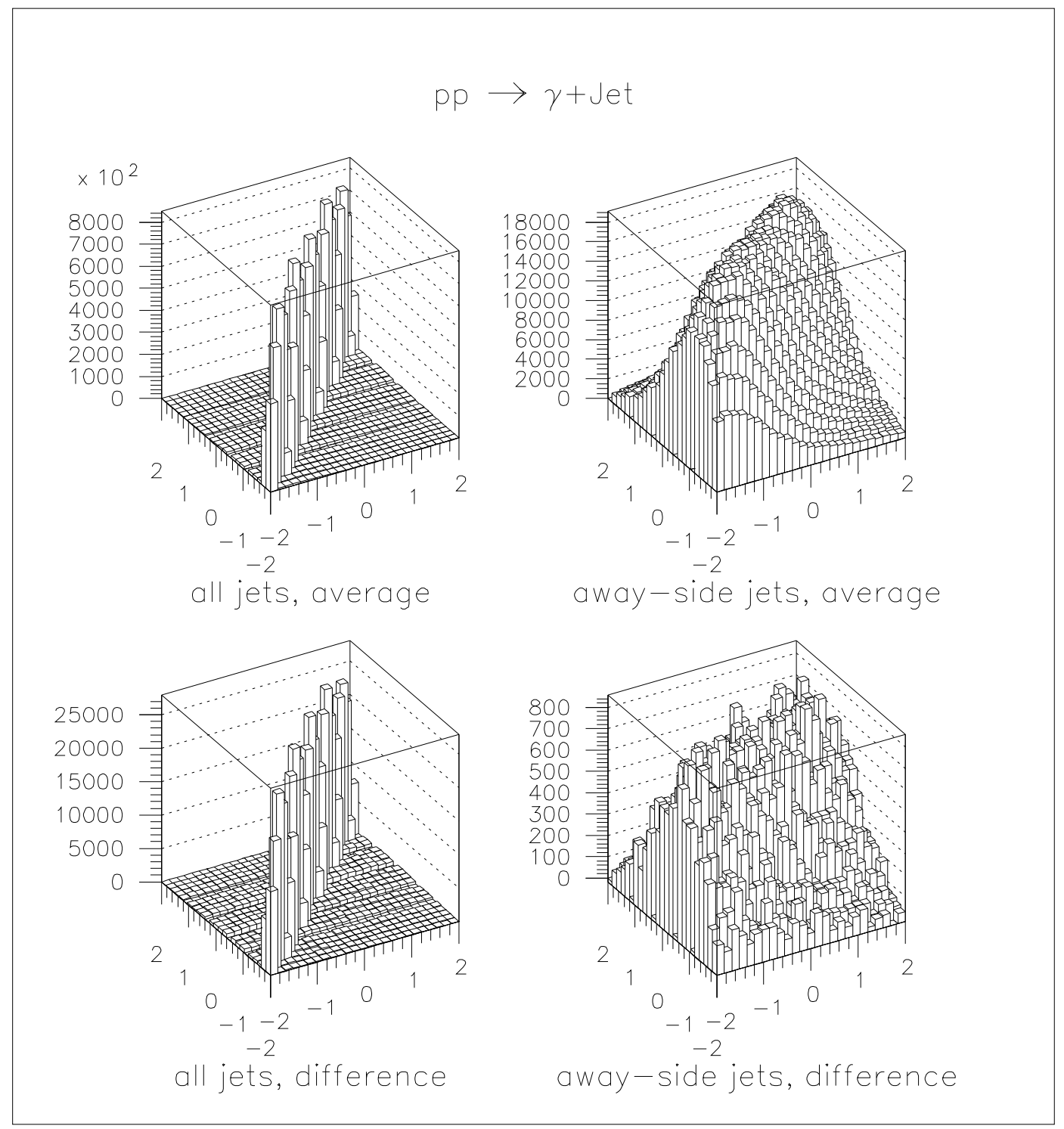

Figure 13: Histogramms for the rapidity distribution of jet events. The photon rapidity $\eta^{\gamma}$ is plotted to the backward-right, the jet-rapidity $\eta^{\text {Jet }}$ is plotted to the backward-left, both in the range from -2 to 2 . Spin average and spin difference are compared for all jets and away-side jets. top: spin average bottom: spin difference left: all jets right: away-side jets only 


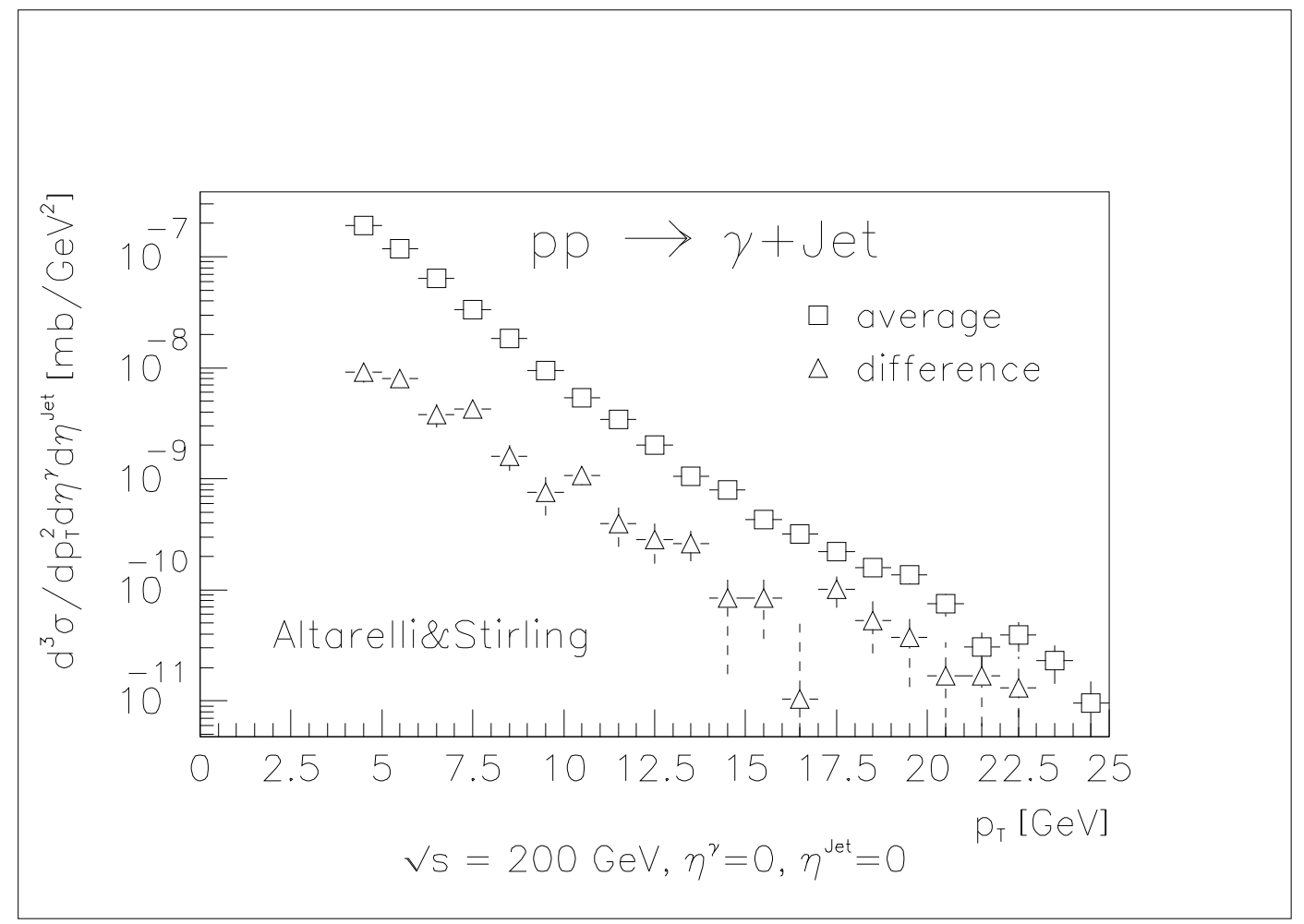

Figure 14: Cross section for prompt- $\gamma$ production with an away-side jet for the Altarelli\&Stirling parametrisation 


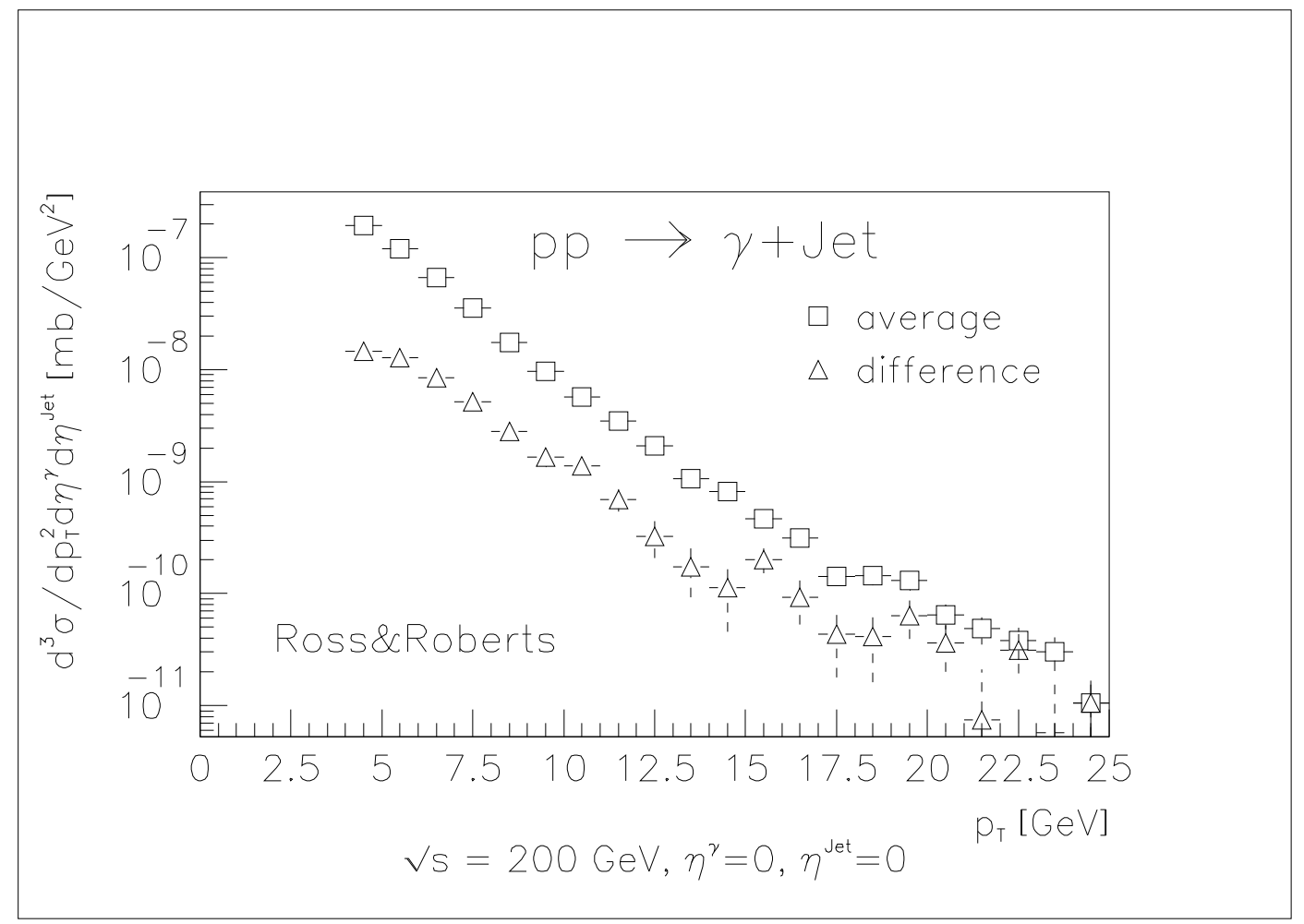

Figure 15: Cross section for prompt- $\gamma$ production with an away-side jet for the Ross\&Roberts parametrisation 


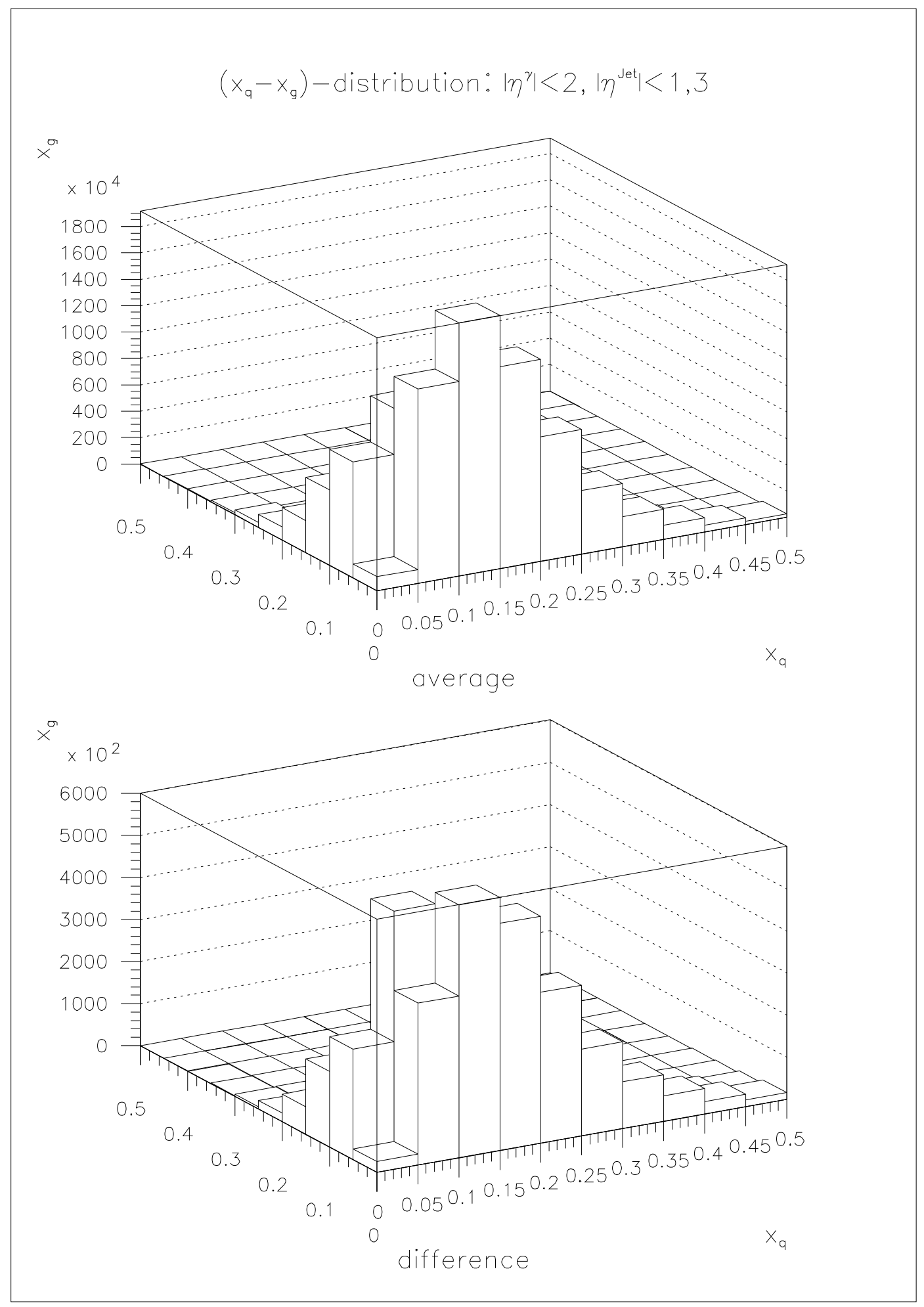

Figure 16: Simulated $\left(x_{q}-x_{g}\right)$ distribution for the STAR detector with end caps. $x_{q}$ is plotted to the right, $x_{g}$ to the left backward. top: spin average bottom: spin difference 


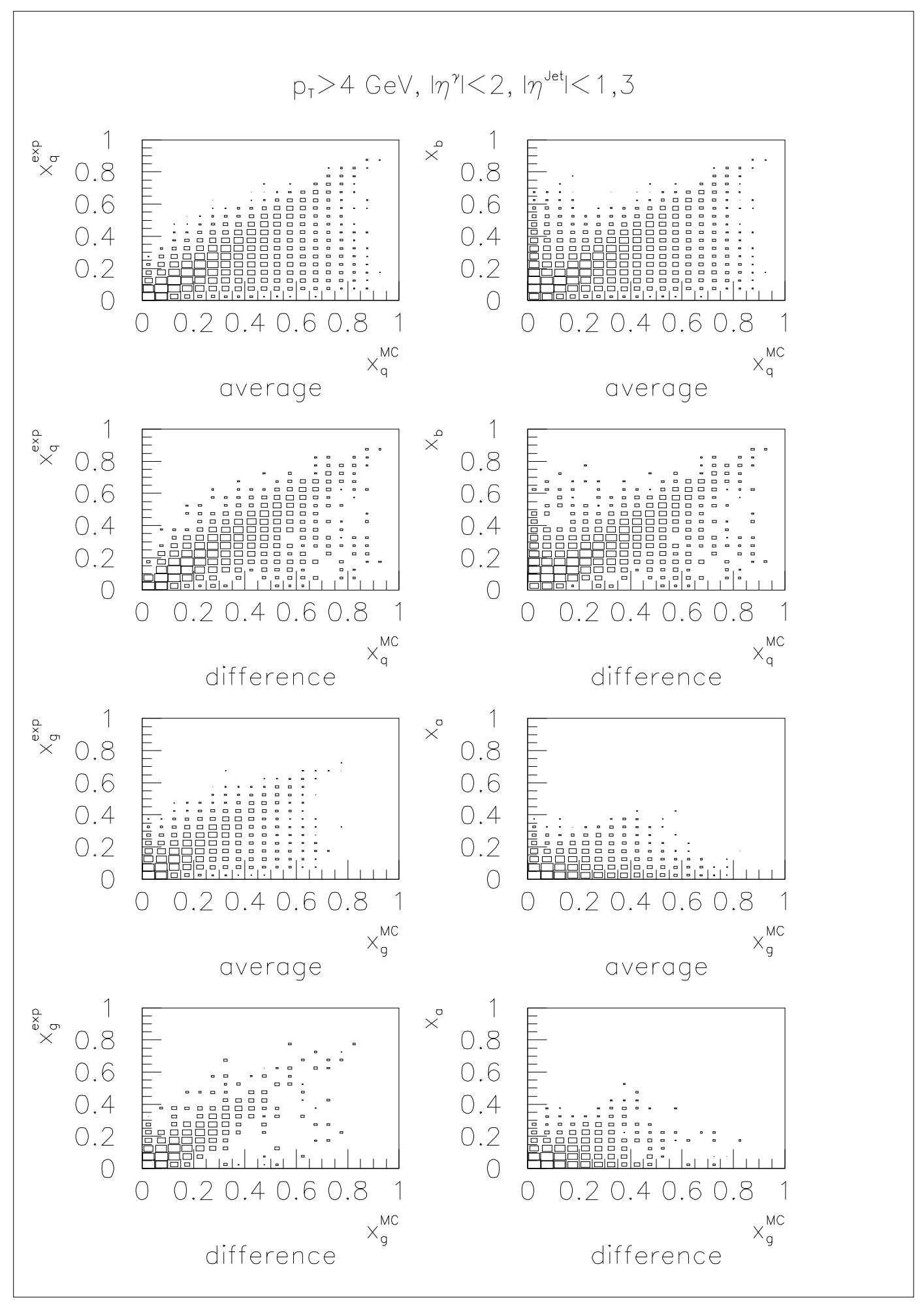

Figure 17: $x$ reconstruction for quarks and gluons, idealy $x_{q}^{\mathrm{MC}}$ should be strictly correlated with $x_{q}^{\exp }$ and $x_{b}$ and $x_{g}^{\mathrm{MC}}$ with $x_{g}^{\mathrm{exp}}$ and $x_{a}$. The areas of the rectangles are proportional to the logarithm of the counts.
1.+2. line: quark
3.+4. line: gluons
left: $x_{M C}$ vs. $x_{\exp }$

1.+3. line: spin average $2 .+4$. line: spin difference right: $x_{a / b}$ vs. $x_{g / q}$ 


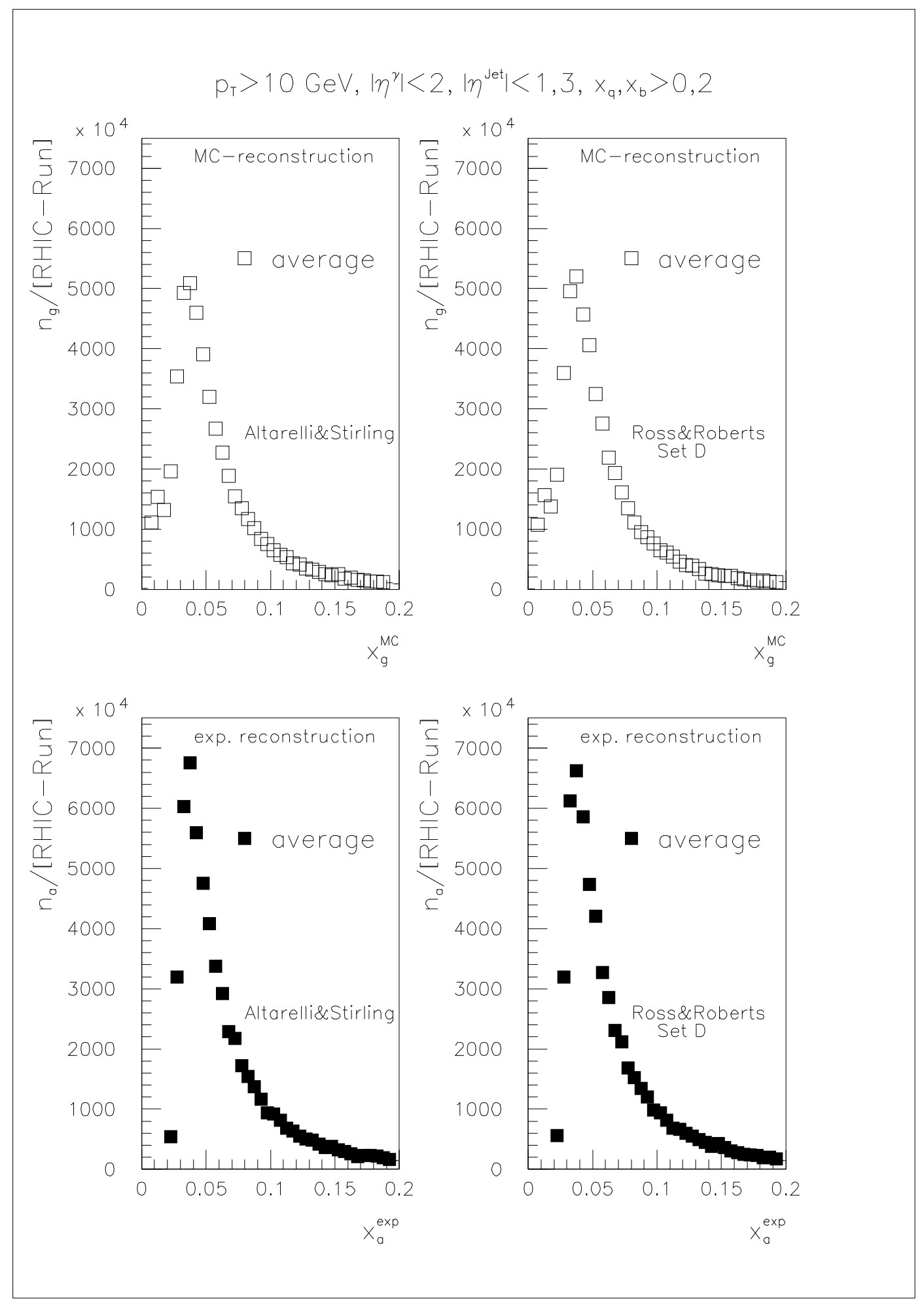

Figure 18: Reconstructed spin averaged $x_{g}$-distributions. MCreconstraction implies that cuts on $x_{q}$ were applied while for 'experimental reconstruction' these cuts were imposed on $x_{b}$.

top: $M C$ reconstruction

bottom: Exp. reconstruction left: Altarelli\&Stirling

rechts: Ross\&Roberts set $D$ 


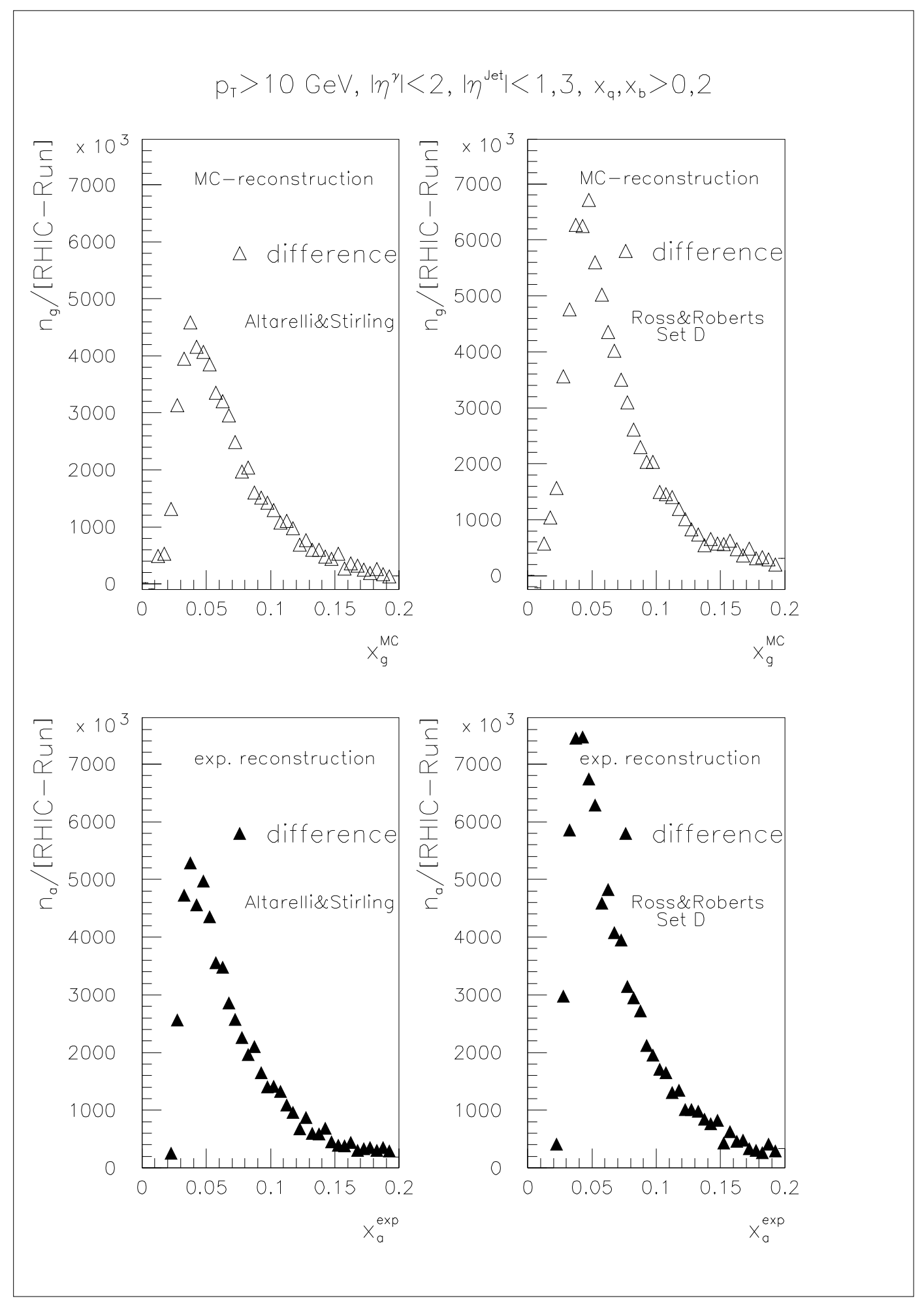

Figure 19: Same as figure $\mathbf{1 8}$ for the spin difference 


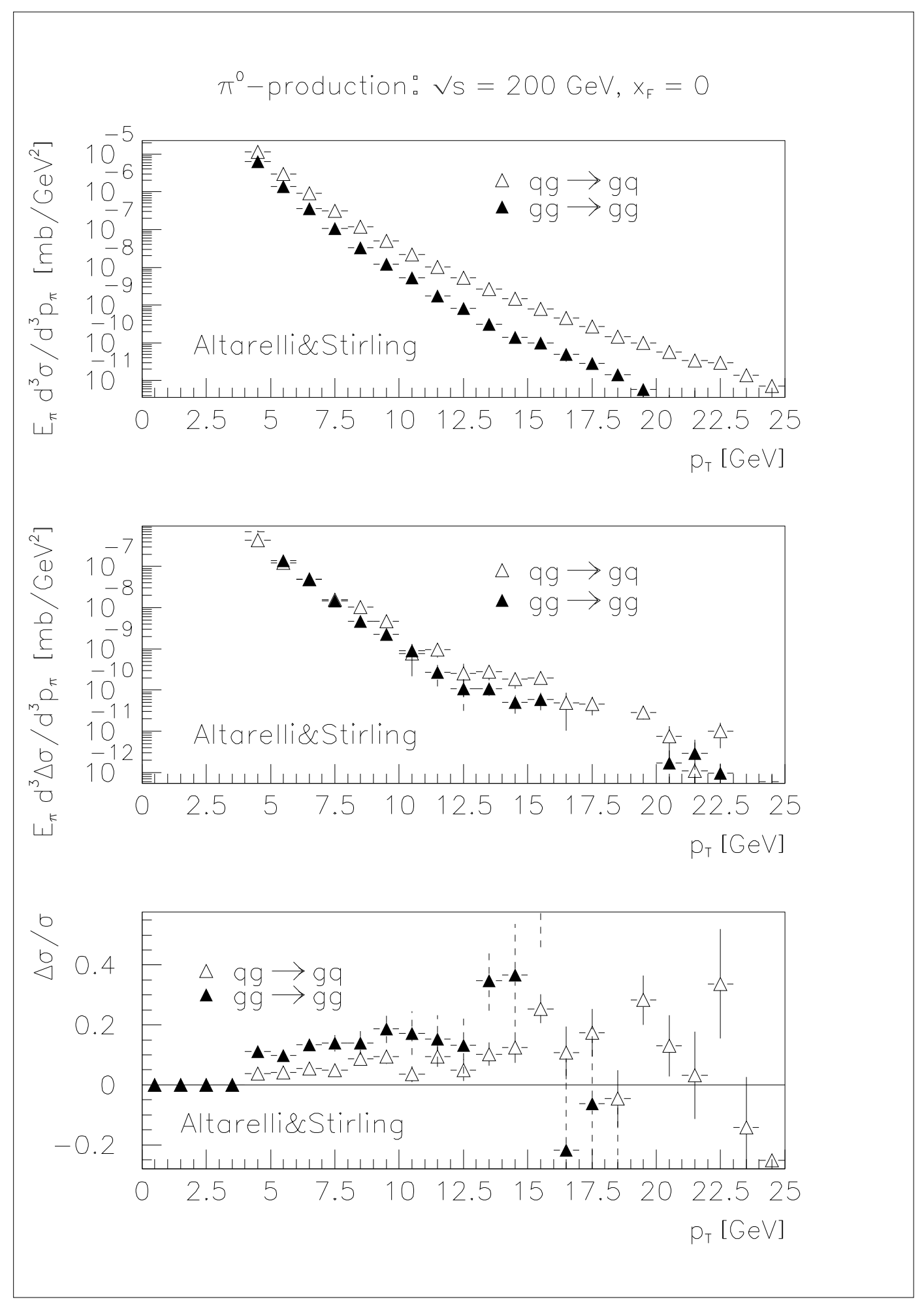

Figure 20: $\pi^{0}$ production to the hard processes $q g \rightarrow g q$ and $g g \rightarrow g g$ top: spin average middle: spin difference bottom: asymmetry 


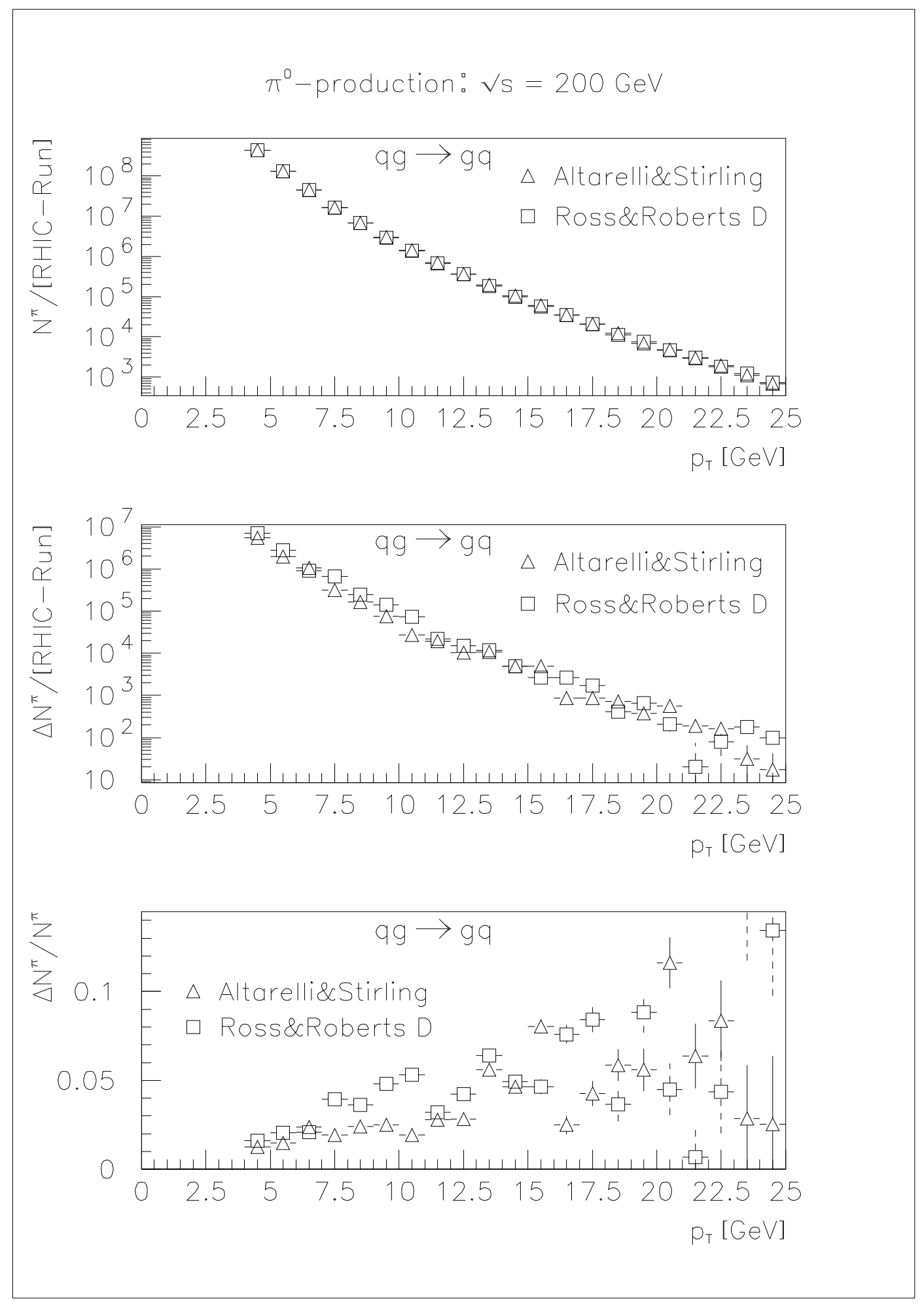

Figure 21: $\pi^{0}$ production: comparison of the results for the Altarelli\&Stirling and the Ross\&Roberts D parametrisation top: spin average middle: spin difference bottom: asymmetry 